\title{
Blind Multiuser Detection by Kurtosis Maximization for Asynchronous Multirate DS/CDMA Systems
}

\author{
Chun-Hsien Peng, Chong-Yung Chi, and Chia-Wen Chang \\ Department of Electrical Engineering and Institute of Communications Engineering, National Tsing Hua University, \\ Hsinchu 30043, Taiwan
}

Received 9 January 2006; Revised 7 July 2006; Accepted 16 July 2006

\begin{abstract}
Chi et al. proposed a fast kurtosis maximization algorithm (FKMA) for blind equalization/deconvolution of multiple-input multiple-output (MIMO) linear time-invariant systems. This algorithm has been applied to blind multiuser detection of singlerate direct-sequence/code-division multiple-access (DS/CDMA) systems and blind source separation (or independent component analysis). In this paper, the FKMA is further applied to blind multiuser detection for multirate DS/CDMA systems. The ideas are to properly formulate discrete-time MIMO signal models by converting real multirate users into single-rate virtual users, followed by the use of FKMA for extraction of virtual users' data sequences associated with the desired user, and recovery of the data sequence of the desired user from estimated virtual users' data sequences. Assuming that all the users' spreading sequences are given a priori, two multirate blind multiuser detection algorithms (with either a single receive antenna or multiple antennas), which also enjoy the merits of superexponential convergence rate and guaranteed convergence of the FKMA, are proposed in the paper, one based on a convolutional MIMO signal model and the other based on an instantaneous MIMO signal model. Some simulation results are then presented to demonstrate their effectiveness and to provide a performance comparison with some existing algorithms.
\end{abstract}

Copyright (C) 2006 Chun-Hsien Peng et al. This is an open access article distributed under the Creative Commons Attribution License, which permits unrestricted use, distribution, and reproduction in any medium, provided the original work is properly cited.

\section{INTRODUCTION}

Direct-sequence/code-division multiple access (DS/CDMA) has been widely used in multiuser cellular wireless communications (e.g., 2G, 3G, and ultra-wideband systems) due to efficient spectrum utilization, release from frequency management, low mobile station's transmit power through power control [1], and high multipath resolution, and so forth [2$8]$. With growing demands for multimedia services in wireless communication systems, there has been a need to provide a platform of high-speed multirate for the transmission of image, video, voice, and data such as variable chip rate (VCR), variable processing gain (VPG) (which is also called variable sequence length), and multicode (MC) DS/CDMA systems [9-17]. For VCR systems, each user uses a spreading sequence of the same length (i.e, different rate users use different chip rates), resulting in that the available bandwidth is not fully used by the low-rate users, whereas the VPG and MC systems avoid this problem. For VPG systems, users with different data rates are accommodated over the same bandwidth (and so a constant chip rate for each user) with the assignment of spreading sequences of different lengths. For MC systems, high-rate users are accommodated by multiplexing their data sequences onto several commonrate data sequences (virtual users) through subsampling, and then a distinct spreading sequence is assigned to each virtual user, and these spreading signals are superimposed before transmission $[12,13]$. The VPG and MC access schemes have been adopted for $3 G$ wireless communication systems $[4,5]$.

In addition to additive white Gaussian noise, it is well known that multiple-access interference (MAI) due to multiple users sharing the same channel and intersymbol interference (ISI) resulting from multipath between the transmitter and receiver are the two major problems encountered in the receiver design of DS/CDMA systems [2, 3, 6-8]. Therefore, a number of single-user (or multiuser) detection algorithms for the efficient suppression of MAI and ISI to improve system performance have been reported for single-rate (or multirate) DS/CDMA systems in the open literature. Conventional detection algorithms (i.e., single-user detection algorithms), where the interfering signals are modeled as noise, are sensitive to the near-far problem such as the RAKE receiver and the matched filter $[2,3,18]$. Thus, many multiuser detection algorithms, where the MAI is explicitly modeled as a part of the signal model, have been proposed for 
single-rate DS/CDMA systems [2, 3, 6-8, 18-25] and for multirate DS/CDMA systems [9-17].

Optimum receivers such as nonlinear maximum likelihood detectors $[2,3,17,18]$ have been reported that are near-far resistant, but their computational complexity grows rapidly with the number of active users. To overcome this drawback, some suboptimal linear detectors with lower computational complexity have been reported such as decorrelating detector $[2,3,18]$, which completely suppresses the unwanted users at the expense of noise enhancement, and minimum mean-square-error (MMSE) detector $[2,3,16,18]$, which performs as the decorrelating detector when noise variance approaches zero. However, the nonblind decorrelating detector and MMSE detector require the channel information estimated through using training sequences or pilot signals in advance resulting in reduced spectral efficiency. Therefore, a blind multiuser detector only using received signals (with no need of training sequences) is preferable.

For multirate DS/CDMA systems, discrete-time multiple-input multiple-output (MIMO) signal models can be formulated through chip-rate sampling of the received continuous-time signal followed by polyphase decomposition. There have been a number of blind MIMO channel identification, equalization (or deconvolution), and beamforming approaches applied to DS/CDMA systems for blind multiusr detection. Tsatsanis et al. [11] and Tsatsanis and $\mathrm{Xu}$ [19] proposed a minimum variance (MV) receiver, which is near-far resistant, to estimate the desired user's symbol sequence based on an instantaneous MIMO signal model. Based on a convolutional MIMO signal model, some subspace-based algorithms [20, 21] were reported for estimation of multipath channels of DS/CDMA systems followed by the design of detection algorithms. Usually, singular value decomposition of correlation matrices with huge dimension must be performed by subspace-based methods, and therefore their practical use is limited due to large computational complexity. On the other hand, Ma and Tugnait's code-constrained inverse filter criteria (CC-IFC) algorithm [13] and $\mathrm{Xu}$ and Liu's code-constrained constant modulus algorithm [14], which are based on the convolutional MIMO signal model, are effective but may not be very computationally efficient.

Recently, Chi et al. [6-8] and Chi and Chen [26] proposed a fast kurtosis maximization algorithm (FKMA) for blind multiuser detection of single-rate DS/CDMA systems and some other applications such as blind beamforming and blind source separation (or independent component analysis). In this paper, the FKMA is further considered for blind multiuser detection of multirate DS/CDMA systems equipped with a single antenna or multiple antennas. With all the users' spreading sequences known a priori, two multirate blind multiuser detection algorithms (BMDAs) using FKMA are proposed, which also enjoy the merits of superexponential convergence rate and guaranteed convergence of the FKMA [7, 26, 27], one, referred to as Algorithm 1, based on a convolutional MIMO signal model and the other, referred to as Algorithm 2, based on an instantaneous MIMO signal model.
The remaining parts of the paper are organized as follows. Section 2 presents the two discrete-time MIMO signal models used by Algorithms 1 and 2, respectively, for the case of single receive antenna. Sections 3 and 4 present Algorithms 1 and 2, respectively, for the estimation of the desired user's data sequence. Section 5 presents how Algorithms 1 and 2 can be applied to the case of multiple receive antennas. Then, some simulation results are provided to support the effectiveness of the proposed algorithms in Section 6. Finally, some conclusions are drawn in Section 7.

\section{MIMO SIGNAL MODELS}

Consider an asynchronous VPG system with a single receive antenna, a constant chip rate $R$ for all the users, and $G$ groups of users. Assume that group $i$ consists of $K_{i}$ users each sharing the same data rate $R_{i}$, where $R_{i} \neq R_{j}$ for all $i \neq j$. For notational clarity, independent variables " $n$ " and " $k$ " are used to denote symbol index and chip index, respectively, in all the discrete-time signals or channels throughout the paper. For ease of later use, some notations are defined as follows:

*: $\quad$ convolution operation of discrete-time

$E\{\cdot\}: \quad$ expectation operator,

$\|\cdot\|: \quad$ Euclidean norm of vectors or matrices,

$\mathbf{0}_{p}$ : $\quad p \times 1$ zero vector,

Superscript “ $*$ ": complex conjugation,

Superscript " $T$ ": transpose of vectors or matrices, Superscript " $H$ ": $\begin{aligned} & \text { complex conjugate transpose (Hermitian) } \\ & \text { of vectors or matrices, }\end{aligned}$

$P_{i}: \quad\left(=R / R_{i}\right)$ spreading factor of users in

group $i$

$P: \quad\left(=\max _{i}\left\{P_{i}\right\}\right)$ maximum spreading factor,

$N_{i}: \quad\left(=P / P_{i}\right)$ number of "virtual users" (defined by (3) below) associated with each user in group $i$,

$K:$

$\left(=\sum_{i=1}^{G} K_{i}\right)$ total number of users,

$\mathcal{K}:$ $\left(=\sum_{i=1}^{G} K_{i} N_{i}\right)$ total number of virtual users,

$u_{i j}[n]:$

$c_{i j}[k]:$

$g_{i j}(t):$

symbol sequence of user $j$ in group $i$, spreading sequence associated with $u_{i j}[n]$,

channel impulse response associated with $u_{i j}[n]$ including the transmitter filter (chip waveform), multipath channel, and receiver filter,

$\operatorname{cum}\left\{x_{1}, x_{2}, \quad\right.$ fourth-order joint cumulant of random

$\left.x_{3}, x_{4}\right\}: \quad$ variables $x_{1}, x_{2}, x_{3}$, and $x_{4}$,

$C_{4}\{x\}=$

$\operatorname{cum}\left\{x_{1}=\right.$

$x, x_{2}=x, x_{3}=$

kurtosis of random variable $x$. 
The received baseband continuous-time signal $y(t)$ in multirate form can be expressed as follows [10-13]:

$$
y(t)=\sum_{i=1}^{G} \sum_{j=1}^{K_{i}} y_{i j}(t)+w(t)
$$

where

$$
y_{i j}(t)=\sum_{k=-\infty}^{\infty} \sum_{n=-\infty}^{\infty} u_{i j}[n] c_{i j}\left[k-n P_{i}\right] g_{i j}\left(t-k T_{c}\right)
$$

is the received baseband continuous-time signal from user $j$ of group $i, T_{c}$ denotes chip duration, and $w(t)$ is white Gaussian noise. Let $u_{i j}^{(l)}[n]$ denote the symbol sequence of the $l$ th virtual user associated with $u_{i j}[n]$ defined as

$$
u_{i j}^{(l)}[n]=u_{i j}\left[n N_{i}+l-1\right], \quad l=1,2, \ldots, N_{i},
$$

and let $c_{i j}^{(l)}[k]$ denote the associated spreading sequence with length equal to $P$ defined as

$$
c_{i j}^{(l)}[k]= \begin{cases}c_{i j}\left[k-(l-1) P_{i}\right], & \text { for }(l-1) P_{i} \leq k \leq l P_{i}-1, \\ 0, & \text { otherwise } .\end{cases}
$$

Then $y(t)$ given by (1) in multirate form can also be converted into a single-rate form as follows:

$$
y(t)=\sum_{i=1}^{G} \sum_{j=1}^{K_{i}} \sum_{l=1}^{N_{i}} \sum_{n=-\infty}^{\infty} u_{i j}^{(l)}[n] h_{i j}^{(l)}\left(t-n P T_{c}\right)+w(t),
$$

where

$$
h_{i j}^{(l)}(t)=\sum_{k=-\infty}^{\infty} c_{i j}^{(l)}[k] g_{i j}\left(t-k T_{c}\right), \quad l=1,2, \ldots, N_{i},
$$

is the effective signature waveform associated with the $l$ th virtual user's symbol sequence $u_{i j}^{(l)}[n]$.

The received discrete-time signal $y[k]$ in single-rate form can be obtained through chip-rate sampling of the received continuous-time signal $y(t)$ specified by (5) as follows:

$$
y[k]=y\left(k T_{c}\right)=\sum_{i=1}^{G} \sum_{j=1}^{K_{i}} \sum_{l=1}^{N_{i}} \sum_{n=-\infty}^{\infty} u_{i j}^{(l)}[n] h_{i j}^{(l)}[k-n P]+w[k],
$$

where $w[k]=w\left(k T_{c}\right)$ and

$$
h_{i j}^{(l)}[k]=h_{i j}^{(l)}\left(k T_{c}\right)=\sum_{\tau=0}^{d_{i j}} c_{i j}^{(l)}[k-\tau] g_{i j}[\tau]
$$

is the effective signature sequence associated with the $l$ th virtual user where $g_{i j}[k]=g_{i j}\left(k T_{c}\right)$ is the discrete-time multipath channel (FIR channel) of order equal to $d_{i j} \leq$ $\min _{i}\left\{P_{i}\right\}$ (a widely used assumption about the channel order in most asynchronous DS/CDMA channels $[6,8,11,19,22])$. Note that the channel impulse response $g_{i j}[k]$ associated with $u_{i j}^{(l)}[n]$ is the same for all $l$. A discrete-time convolutional MIMO signal model and a discrete-time instantaneous MIMO signal model are presented next, respectively.

\subsection{Convolutional MIMO signal model}

Collecting $P$ chip-rate samples of $y[k]$ given by (7) into a $P \times 1$ column vector (polyphase decomposition), one can obtain a discrete-time convolutional (or memory) MIMO signal model $[6-8,12-14,16,17,24]$ as follows:

$$
\begin{aligned}
\mathbf{y}[n] & =(y[n P], y[n P+1], \ldots, y[n P+P-1])^{T} \\
& =\sum_{i=1}^{G} \sum_{j=1}^{K_{i}} \sum_{l=1}^{N_{i}} \mathbf{y}_{i j}^{(l)}[n]+\mathbf{w}[n] \\
& =\mathbf{H}[n] * \mathbf{u}[n]+\mathbf{w}[n]=\sum_{n_{1}=0}^{1} \mathbf{H}\left[n_{1}\right] \mathbf{u}\left[n-n_{1}\right]+\mathbf{w}[n],
\end{aligned}
$$

where $\mathbf{w}[n]$ is a white Gaussian noise vector,

$$
\begin{gathered}
\mathbf{u}[n]=\left(u_{11}^{(1)}[n], \ldots, u_{11}^{\left(N_{1}\right)}[n], u_{12}^{(1)}[n], \ldots,\right. \\
\left.u_{12}^{\left(N_{1}\right)}[n], \ldots, u_{G K_{G}}^{(1)}[n], \ldots, u_{G K_{G}}^{\left(N_{G}\right)}[n]\right)^{T}, \\
\mathbf{y}_{i j}^{(l)}[n]=\mathbf{h}_{i j}^{(l)}[n] * u_{i j}^{(l)}[n]=\sum_{n_{1}=0}^{1} \mathbf{h}_{i j}^{(l)}\left[n_{1}\right] u_{i j}^{(l)}\left[n-n_{1}\right], \\
\mathbf{H}[n]=\left(\mathbf{h}_{11}^{(1)}[n], \ldots, \mathbf{h}_{11}^{\left(N_{1}\right)}[n], \mathbf{h}_{12}^{(1)}[n], \ldots,\right. \\
\left.\mathbf{h}_{12}^{\left(N_{1}\right)}[n], \ldots, \mathbf{h}_{G K_{G}}^{(1)}[n], \ldots, \mathbf{h}_{G K_{G}}^{\left(N_{G}\right)}[n]\right),
\end{gathered}
$$

in which

$$
\mathbf{h}_{i j}^{(l)}[n]=\left(h_{i j}^{(l)}[n P], h_{i j}^{(l)}[n P+1], \ldots, h_{i j}^{(l)}[n P+P-1]\right)^{T} .
$$

Note that, in the convolutional MIMO signal model given by (9), $\mathbf{H}[n]$ is a $P \times \mathcal{K}$ impulse response matrix formed by all the effective signature vectors $\mathbf{h}_{i j}^{(l)}[n]$ 's (see $(13)$ ), $\mathbf{u}[n]$ is a $\mathcal{K} \times 1$ signal vector composed of all the virtual users' signals (or sources) $u_{i j}^{(l)}[n]$ 's (see $\left.(3)\right)$.

\subsection{Instantaneous MIMO signal model}

For ease of later use, define

$$
\begin{gathered}
q=\max _{i j}\left\{d_{i j}\right\} \leq \min _{i}\left\{P_{i}\right\} \\
\leq P_{i} \quad\left(d_{i j} \text { is the order of the channel } g_{i j}[k]\right), \\
\mathbf{g}_{i j}=\left(g_{i j}[0], g_{i j}[1], \ldots, g_{i j}\left[d_{i j}\right], \mathbf{0}_{\left(q-d_{i j}\right)}^{T}\right)^{T}, \\
u_{i j, m}^{(l)}[n]=u_{i j}^{(l)}[n-m], \quad m=-1,0,1, \\
\mathbf{c}_{i j}^{(l)}=\left(c_{i j}^{(l)}[0], c_{i j}^{(l)}[1], \ldots, c_{i j}^{(l)}[P-1]\right)^{T},
\end{gathered}
$$

$\mathrm{h}_{i j, m}^{(l)}$

$$
= \begin{cases}\left(\mathbf{0}_{P}^{T}, h_{i j}^{(l)}[0], h_{i j}^{(l)}[1], \ldots, h_{i j}^{(l)}[q-1]\right)^{T}, & m=-1, \\ \left(h_{i j}^{(l)}[0], h_{i j}^{(l)}[1], \ldots, h_{i j}^{(l)}[P+q-1]\right)^{T}, & m=0, \\ \left(h_{i j}^{(l)}[P], h_{i j}^{(l)}[P+1], \ldots, h_{i j}^{(l)}[P+q-1], \mathbf{0}_{P}^{T}\right)^{T}, & m=1 .\end{cases}
$$


Note that $q$ (see (14)) is the maximum channel order over all the users' chip-rate channels, the $(q+1) \times 1$ vector $\mathbf{g}_{i j}$ (see $(15))$ is the channel vector associated with $u_{i j}[n]$, and the $P \times 1$ vector $\mathbf{c}_{i j}^{(l)}$ (see (17)) is the spreading code vector associated with $u_{i j}^{(l)}[n]$, and that $u_{i j, m}^{(l)}[n]$ (see (16)) for each $m \neq 0$ is a symbol sequence (with one symbol time delay or advance) associated with the same virtual user's symbol sequence $u_{i j}^{(l)}[n]$ for $m=0$, and $\mathrm{h}_{i j, m}^{(l)}$ (see (18)) is the associated signature vector (see the instantaneous MIMO signal model of $y[n]$ given by (24) below). It can be easily shown, by (8) and (18), that

$$
\mathbf{h}_{i j, m}^{(l)}=\mathbf{C}_{i j, m}^{(l)} \mathbf{g}_{i j},
$$

where $\mathbf{g}_{i j}$ is given by (15) and

$$
\mathbf{C}_{i j, m}^{(l)}= \begin{cases}\left(\widetilde{\mathbf{c}}_{i j, 0}^{(l)}, \widetilde{\mathbf{c}}_{i j, 1}^{(l)}, \ldots, \tilde{\mathbf{c}}_{i j, q}^{(l)}\right), & m=-1, \\ \left(\mathbf{c}_{i j, 0}^{(l)}, \mathbf{c}_{i j, 1}^{(l)}, \ldots, \mathbf{c}_{i j, q}^{(l)}\right), & m=0, \\ \left(\overline{\mathbf{c}}_{i j, 0}^{(l)}, \overline{\mathbf{c}}_{i j, 1}^{(l)}, \ldots, \overline{\mathbf{c}}_{i j, q}^{(l)}\right), & m=1,\end{cases}
$$

in which

$$
\begin{gathered}
\tilde{\mathbf{c}}_{i j, r}^{(l)}=\left(\mathbf{0}_{P}^{T}, c_{i j, r}^{(l)}[0], c_{i j, r}^{(l)}[1], \ldots,\right. \\
\left.c_{i j, r}^{(l)}[q-1]\right)^{T}, \quad(P+q) \times 1 \text { vector }, \\
\mathbf{c}_{i j, r}^{(l)}=\left(c_{i j, r}^{(l)}[0], c_{i j, r}^{(l)}[1], \ldots, c_{i j, r}^{(l)}[P+q-1]\right)^{T} \\
=\left(\mathbf{0}_{r}^{T},\left(\mathbf{c}_{i j}^{(l)}\right)^{T}, \mathbf{0}_{(q-r)}^{T}\right)^{T}, \quad(P+q) \times 1 \text { vector }, \\
\overline{\mathbf{c}}_{i j, r}^{(l)}=\left(c_{i j, r}^{(l)}[P], c_{i j, r}^{(l)}[P+1], \ldots,\right. \\
\left.c_{i j, r}^{(l)}[P+q-1], \mathbf{0}_{P}^{T}\right)^{T}, \quad(P+q) \times 1 \text { vector. }
\end{gathered}
$$

Furthermore, collecting $(P+q)$ chip-rate samples of $y[k]$ given by $(7)$ into a $(P+q) \times 1$ column vector (polyphase decomposition), then a discrete-time instantaneous (or memoryless) MIMO signal model can be obtained as follows $[11,19,25]$ :

$$
\begin{aligned}
\mathrm{y}[n] & =(y[n P], y[n P+1], \ldots, y[n P+P+q-1])^{T} \\
& =\sum_{i=1}^{G} \sum_{j=1}^{K_{i}} \sum_{l=1}^{N_{i}} \sum_{m=-1}^{1} \mathrm{~h}_{i j, m}^{(l)} u_{i j, m}^{(l)}[n]+\mathrm{w}[n]=\mathscr{H} \mathrm{u}[n]+\mathrm{w}[n],
\end{aligned}
$$

where

$$
\begin{aligned}
\mathscr{H}= & \left(\mathrm{h}_{11,-1}^{(1)}, \mathrm{h}_{11,0}^{(1)}, \mathrm{h}_{11,1}^{(1)}, \ldots, \mathrm{h}_{11,-1}^{\left(N_{1}\right)}, \mathrm{h}_{11,0}^{\left(N_{1}\right)},\right. \\
& \left.\mathrm{h}_{11,1}^{\left(N_{1}\right)}, \ldots, \mathrm{h}_{G K_{G},-1}^{\left(N_{G}\right)}, \mathrm{h}_{G K_{G}, 0}^{\left(N_{G}\right)}, \mathrm{h}_{G K_{G}, 1}^{\left(N_{G}\right)}\right), \\
\mathrm{u}[n]=( & u_{11,-1}^{(1)}[n], u_{11,0}^{(1)}[n], u_{11,1}^{(1)}[n], \ldots, \\
& u_{11,-1}^{\left(N_{1}\right)}[n], u_{11,0}^{\left(N_{1}\right)}[n], u_{11,1}^{\left(N_{1}\right)}[n], \ldots, \\
& \left.u_{G K_{G},-1}^{\left(N_{G}\right)}[n], u_{G K_{G}, 0}^{\left(N_{G}\right)}[n], u_{G K_{G}, 1}^{\left(N_{G}\right)}[n]\right)^{T},
\end{aligned}
$$

and $\mathrm{w}[n]$ is a $(P+q) \times 1$ white Gaussian noise vector. Note that $\mathscr{H}$ is a $(P+q) \times(3 \mathcal{K})$ MIMO channel matrix composed of $3 \mathcal{K}$ column vectors $\mathrm{h}_{i j, m}^{(l)}$ 's and $\mathrm{u}[n]$ is a $(3 \mathcal{K}) \times 1$ vector composed of $3 \mathcal{K}$ sources $u_{i j, m}^{(l)}[n]$ 's.

Three worthy remarks about the preceding convolutional MIMO signal model and instantaneous MIMO signal model are as follows.

(R1) One can observe, by (4) and (17) to (23), that $\mathrm{h}_{i j,-1}^{(l)}=\mathbf{0}_{P+q}$ for all $2 \leq l \leq N_{i}$ and $\mathrm{h}_{i j, 1}^{(l)}=\mathbf{0}_{P+q}$ for $1 \leq l \leq N_{i}-1$ for the VPG system. Therefore, by removing these zero column vectors in $\mathscr{H}$, the channel matrix $\mathscr{H}$ reduces to a $(P+q) \times(\mathcal{K}+2 K)$ matrix instead of a $(P+q) \times(3 \mathcal{K})$ matrix, and then the associated signal vector $\mathrm{u}[n]$ consists of only $(\mathcal{K}+2 K)(\leq 3 \mathcal{K})$ components out of all the $3 \mathcal{K}$ sources $u_{i j, m}^{(l)}[n]$ 's.

(R2) The inputs (sources) $\mathrm{u}[n]$ (see (26)) (of the instantaneous MIMO signal model $\mathrm{y}[n]$ given by (24)) consist of not only $u_{i j, 0}^{(l)}[n]=u_{i j}^{(l)}[n]$ but also the ISI related sources, that is, $u_{i j, m}^{(l)}[n]=u_{i j}^{(l)}[n-m]$ for $m \neq 0$ (see (16)), and therefore not all the source signals in $\mathrm{u}[n]$ are mutually statistically independent random processes because $E\left\{\mathrm{u}[n] \mathrm{u}^{H}[n-1]\right\}$ is a nondiagonal and nonzero matrix (i.e., $\mathrm{u}[n]$ itself is not an independent vector process).

(R3) As mentioned in the introduction section, for an asynchronous MC system [9, 12, 13], a high-rate symbol sequence $u_{i j}[n]$ can be converted into $N_{i}$ symbol subsequences $u_{i j}^{(l)}[n]$ 's (i.e., $N_{i}$ virtual users), as defined by (3), each assigned a distinct spreading sequence $c_{i j}^{(l)}[k]$ (with the same processing gain $\left.P=R / \min _{i}\left\{R_{i}\right\}\right)$. All the $N_{i}$ virtual users' spreading signals are superimposed prior to transmission. Following the same modeling procedure of the VPG system, one can also obtain a discrete-time convolutional MIMO signal model $\mathbf{y}[n]$ and an instantaneous MIMO signal model $\mathrm{y}[n]$ which have exactly the same forms given by (9) and (24), respectively, for the MC system except that $\mathrm{h}_{i j, m}^{(l)} \neq \mathbf{0}_{P+q}$ for all $i, j, m$, and $l$, and $d_{i j} \leq P$ (rather than $d_{i j} \leq \min _{i}\left\{P_{i}\right\}$ as in the VPG system).

Assume that the user of interest is user 1 in group 1 (i.e., $\left.u_{11}[n]\right)$ for simplicity. Our goal is to design a linear detector to extract $u_{11}[n]$ by processing either $\mathrm{y}[n]$ or $\mathrm{y}[n]$ without training sequences. Next, let us present a multirate BMDA for the VPG or MC system using $\mathbf{y}[n]$ and another multirate BMDA using $\mathrm{y}[n]$ for estimating $u_{11}[n]$ by kurtosis maximization.

\section{MULTIRATE BMDA USING CONVOLUTIONAL MIMO SIGNAL MODEL}

The proposed multirate BMDA using the convolutional MIMO signal model $\mathbf{y}[n]$ given by (9) comprises estimation of all the virtual users' symbol sequences (or source signals) $u_{11}^{(l)}[n], l=1,2, \ldots, N_{1}$, and recovery of the desired user's symbol sequence $u_{11}[n]$ from the obtained estimates $\hat{u}_{11}^{(l)}[n]$ 's.

Some general assumptions for $\mathbf{y}[n]$ specified by (9) are made as follows $[6,8]$. 
(A1) $u_{i j}[n]$ for all $i$ and $j$ are independent identically distributed (i.i.d.) zero-mean non-Gaussian with $C_{4}\left\{u_{i j}[n]\right\} \neq 0$, and statistically independent of $u_{l q}[n]$ for all $(i, j) \neq(l, q)$.

(A2) The $P \times \mathcal{K}$ system $\mathbb{H}(z)$ (z-transform of $\mathbf{H}[n])$ is bounded-input bounded-output stable and of full column rank for all $|z|=1$. Moreover, $P \geq \mathcal{K}$.

(A3) $\mathbf{w}[n]$ is zero-mean Gaussian, and statistically independent of $\mathbf{u}[n]$.

Note that assumption (A1) and (3) imply the following fact.

Fact 1. $u_{i j}^{(l)}[n]$ is a zero-mean non-Gaussian i.i.d. process with $C_{4}\left\{u_{i j}^{(l)}[n]\right\}=C_{4}\left\{u_{i j}[n]\right\} \neq 0$, and meanwhile $u_{i j}^{(l)}[n]$ 's are mutually statistically independent.

\subsection{Extraction of source signals}

Let $e[n]$ be the output of a $P \times 1$ linear equalizer $\mathbf{v}[n]$ (an FIR filter) of length $L$ to be designed, that is,

$$
e[n]=\mathbf{v}^{T}[n] * \mathbf{y}[n]=\sum_{l=0}^{L-1} \mathbf{v}^{T}[l] \mathbf{y}[n-l]=v^{T} \psi[n],
$$

where

$$
\begin{gathered}
v=\left(\mathbf{v}^{T}[0], \mathbf{v}^{T}[1], \ldots, \mathbf{v}^{T}[L-1]\right)^{T}, \\
\psi[n]=\left(\mathbf{y}^{T}[n], \mathbf{y}^{T}[n-1], \ldots, \mathbf{y}^{T}[n-L+1]\right)^{T} .
\end{gathered}
$$

Let

$$
\begin{aligned}
J(e[n]) & =J(v)=\frac{\left|C_{4}\{e[n]\}\right|}{\left(E\left\{|e[n]|^{2}\right\}\right)^{2}} \\
& =\frac{\left.\left|E\left\{|e[n]|^{4}\right\}-2\left(E\left\{|e[n]|^{2}\right\}\right)^{2}-\right| E\left\{e^{2}[n]\right\}\right|^{2} \mid}{\left(E\left\{|e[n]|^{2}\right\}\right)^{2}},
\end{aligned}
$$

which is also the magnitude of normalized kurtosis of $e[n]$ $[6-8,13,28]$.

The iterative FKMA proposed by Chi et al. [6-8] can be used to find the optimum $v$ by maximizing $J(\nu)$ through the following two steps at each iteration.

Step 1. Update $v^{(i)}$ at the ith iteration by

$$
v^{(i)}=\frac{\left(\mathbf{R}_{\psi}^{*}\right)^{-1} \mathbf{d}\left(e^{(i-1)}[n], \psi[n]\right)}{\left\|\left(\mathbf{R}_{\psi}^{*}\right)^{-1} \mathbf{d}\left(e^{(i-1)}[n], \psi[n]\right)\right\|},
$$

where

$$
\begin{aligned}
& \mathbf{R}_{\psi}=E\left\{\psi[n] \psi^{H}[n]\right\}, \\
\mathbf{d}(e[n], \psi[n])= & \operatorname{cum}\left\{e[n], e[n], e^{*}[n], \psi^{*}[n]\right\} \\
= & E\left\{|e[n]|^{2} e[n] \psi^{*}[n]\right\} \\
& -2 E\left\{|e[n]|^{2}\right\} E\left\{e[n] \psi^{*}[n]\right\} \\
& -E\left\{e^{2}[n]\right\} E\left\{e^{*}[n] \psi^{*}[n]\right\} .
\end{aligned}
$$

Then obtain the associated $e^{(i)}[n]=\left(\nu^{(i)}\right)^{T} \psi[n]$.
Step 2. If $J\left(\nu^{(i)}\right)>J\left(v^{(i-1)}\right)$, go to the next iteration; otherwise reupdate $\nu^{(i)}$ through a gradient-type optimization algorithm, that is,

$$
\nu^{(i)}=\nu^{(i-1)}+\left.\rho \frac{\partial J(\nu)}{\partial \nu^{*}}\right|_{\nu=\nu^{(i-1)}},
$$

where $\rho$ is a step size parameter and

$$
\begin{aligned}
\frac{\partial J(\nu)}{\partial \nu^{*}}=2 J(\nu) \cdot & \left\{\frac{1}{C_{4}\{e[n]\}} \cdot \mathbf{d}(e[n], \psi[n])\right. \\
& \left.-\frac{1}{E\left\{|e[n]|^{2}\right\}} \cdot \mathbf{R}_{\psi} \nu\right\}
\end{aligned}
$$

(see [23]) such that $J\left(\nu^{(i)}\right)>J\left(\nu^{(i-1)}\right)$, and then obtain the associated $e^{(i)}[n]=\left(\nu^{(i)}\right)^{T} \psi[n]$.

As stated in $[6,29]$, the FKMA uses the MIMO superexponential algorithm in Step 1 for fast convergence (basically with superexponential rate) which usually happens in most of iterations before convergence, and a gradient-type optimization method in Step 2 for the guaranteed convergence. Therefore, the FKMA is computationally much faster than gradient-type optimization algorithms. Note, from (33), (34), and (35), that $\mathbf{d}\left(e^{i-1}[n], \psi[n]\right)$ required for computing $\partial J(\nu) / \partial \nu^{*}$ (see (35)) in Step 2 has been obtained in Step 1, and the correlation matrix $\mathbf{R}_{\psi}$ is the same at each iteration, indicating simple and straightforward computation for updating $v^{(i)}$ in Step 2.

Under assumptions (A2) and (A3) and Fact 1, the optimum $e[n]$ obtained by FKMA is known to be one of the source signals in $\mathbf{u}[n]$ (except for an unknown scale factor $\alpha_{i j}^{(l)}$ and an unknown time delay $\left.\tau_{i j}^{(l)}\right)$ for SNR $=\infty[6-8]$, and for finite SNR,

$$
e[n]=\widehat{u}_{i j}^{(l)}[n] \simeq \alpha_{i j}^{(l)} u_{i j}^{(l)}\left[n-\tau_{i j}^{(l)}\right],
$$

where the subscripts $i$ as well as $j$ and the superscript $l$ are unknown. However, the unknown $(i, j, l)$ can be efficiently identified using the user identification algorithm (UIA) reported in [8] from all the known spreading sequences $c_{i j}^{(l)}[k]$ 's and the following channel estimate $[6-8,28]$ :

$$
\begin{aligned}
\hat{\mathbf{h}}_{i j}^{(l)}[n] & =\frac{E\left\{\mathbf{y}\left[n_{1}\right] e^{*}\left[n_{1}-n\right]\right\}}{E\left\{\left|e\left[n_{1}\right]\right|^{2}\right\}} \\
& \simeq \frac{1}{\alpha_{i j}^{(l)}} \mathbf{h}_{i j}^{(l)}\left[n+\tau_{i j}^{(l)}\right] \quad(\text { by (9) and (36)). }
\end{aligned}
$$

Assume that $(\hat{i}, \hat{j}, \hat{l})$ is the obtained estimate using the UIA reported in [8]. If $(\hat{i}, \hat{j}, \hat{l})=(1,1, \ell)$, then the desired source estimate $e[n]=\hat{u}_{11}^{(\ell)}[n]$ is obtained, otherwise, one has to update $\mathbf{y}[n]$ by $\mathbf{y}[n]-\widehat{\mathbf{h}}_{i j}^{(l)}[n] * e[n]$ (cancellation or deflation processing) and then repeat the preceding signal processing stage (source extraction using FKMA followed by user identification) until the estimate $\hat{u}_{11}^{(\ell)}[n]$ is obtained (except for an unknown scale factor and an unknown time delay). However, as any other source extraction algorithms 
involving the above multistage successive cancellation procedure, an inevitable disadvantage is given in the following remark.

(R4) A well-designed initial condition for $\mathbf{v}[n]$ reported in [8] usually leads to $(\hat{i}, \hat{j}, \hat{l})=(1,1, \ell)$ at the first stage (without going through deflation processing), otherwise, the estimate $\hat{u}_{11}^{(\ell)}[n]$ obtained at later stage is usually less accurate due to error propagation effects caused by the deflation processing.

\subsection{Recovery of the desired user's symbol sequence}

To obtain $\hat{u}_{11}[n]$ from all the symbol sequence estimates $\hat{u}_{11}^{(l)}[n], l=1,2, \ldots, N_{1}$ (of the desired virtual users), requires the estimation of relative scale factors and relative time delays defined as

$$
\begin{gathered}
\lambda_{11}^{(l)}=\frac{\alpha_{11}^{(1)}}{\alpha_{11}^{(l)}}, \quad l=1,2, \ldots, N_{1}, \\
\Delta_{11}^{(l)}=\tau_{11}^{(1)}-\tau_{11}^{(l)}, \quad l=1,2, \ldots, N_{1},
\end{gathered}
$$

respectively. By (8), (13), and (37), one can infer that

$$
\hat{h}_{i j}^{(l)}[k] \simeq \frac{h_{i j}^{(l)}\left[k+\tau_{i j}^{(l)} P\right]}{\alpha_{i j}^{(l)}}=\frac{c_{i j}^{(l)}[k] * g_{i j}\left[k+\tau_{i j}^{(l)} P\right]}{\alpha_{i j}^{(l)}} .
$$

Note that $c_{i j}^{(l)}[k] * c_{i j}^{(l)}[-k] \simeq \beta_{i} \delta[k]$ since the spreading sequence $c_{i j}^{(l)}[k]$ approximates a pseudonoise sequence where $\beta_{i}=P_{i}$ for the VPG system and $\beta_{i}=P$ for the MC system. Therefore, a chip-rate channel estimate $\hat{g}_{11}^{(l)}[k]$ can be obtained as

$$
\hat{g}_{11}^{(l)}[k]=c_{11}^{(l)}[-k] * \hat{h}_{11}^{(l)}[k] \simeq \frac{\beta_{1} \cdot g_{11}\left[k+\tau_{11}^{(l)} P\right]}{\alpha_{11}^{(l)}},
$$

which is an estimate of the common channel $g_{11}[k]$ associated with $u_{11}^{(l)}[n]$ for all $l$. Then the relative time delays $\Delta_{11}^{(l)}$ with respect to $u_{11}^{(1)}[n]$ can be estimated as follows:

$$
\Delta_{11}^{(l)}=\arg \max _{n}\left\{\left|\sum_{k=-\infty}^{\infty} \hat{g}_{11}^{(1)}[k] \cdot\left(\hat{g}_{11}^{(l)}[k+n P]\right)^{*}\right|\right\} .
$$

On the other hand, let $k_{11}^{(l)}$ denote the peak location of $\hat{g}_{11}^{(l)}[k]$, that is,

$$
k_{11}^{(l)}=\arg \max _{k}\left\{\left|\hat{g}_{11}^{(l)}[k]\right|\right\} .
$$

The parameter $\lambda_{11}^{(l)}$ can be estimated as

$$
\lambda_{11}^{(l)}=\frac{\hat{g}_{11}^{(l)}\left[k_{11}^{(l)}\right]}{\hat{g}_{11}^{(1)}\left[k_{11}^{(1)}\right]} .
$$

By (3), (36), (42), and (44), we obtain the symbol sequence estimate of the desired user (by compensating different time delays $\Delta_{11}^{(l)}$ and amplitude scale factors $\lambda_{11}^{(l)}$ of the symbol estimates of the desired virtual users) as follows.

$$
\hat{u}_{11}[n]=\lambda_{11}^{(l)} \cdot \hat{u}_{11}^{(l)}\left[\tilde{n}-\Delta_{11}^{(l)}\right] \simeq \alpha_{11}^{(1)} u_{11}\left[n-\tau_{11}^{(1)}\right],
$$

where $l=\left(n\right.$ modulo $\left.N_{1}\right)+1$ and $\tilde{n}=(n-l+1) / N_{1}$ (i.e., $n=\tilde{n} N_{1}+l-1$, where $\left.l \in\left\{1,2, \ldots, N_{1}\right\}\right)$. Note that $\alpha_{11}^{(1)}$ and $\tau_{11}^{(1)}$ are the unknown scale factor and time delay in the estimate $\hat{u}_{11}[n]$, respectively.

Let us summarize the resultant multirate BMDA using the convolutional model $\mathbf{y}[n]$, referred to as Algorithm 1, as follows.

Algorithm 1. (S1) As presented in Section 3.1, obtain $\hat{u}_{11}^{(l)}[n]$, $l=1,2, \ldots, N_{1}$, using the FKMA and UIA reported in [8].

(S2) As presented in Section 3.2, obtain $\hat{u}_{11}[n]$ from $\widehat{u}_{11}^{(l)}[n], l=1,2, \ldots, N_{1}$, using (45).

Let us conclude this section with the following two remarks about the proposed Algorithm 1.

(R5) Algorithm 1, which also enjoys the merits of superexponential convergence rate and guaranteed convergence of the FKMA $[6,7,26,27]$ for source extraction, is also applicable to both VPG and MC systems as long as the discrete-time signal vector $\mathrm{y}[n]$ given (9) is obtained.

(R6) Ma and Tugnait's CC-IFC algorithm [13], which uses the convolutional model $\mathrm{y}[n]$ as well, simultaneously estimates all the $u_{11}^{(l)}[n], l=1,2, \ldots, N_{1}$, in "synchronization" (same time delay and scale factor) by minimizing the sum of $-J(e[n])$ and some penalty functions (leading to an extra constraint $L \geq 3$ ). However, the estimates $\hat{u}_{11}^{(l)}[n]$ 's are obtained through using a gradient-type search algorithm (computationally not very efficient) without need of user identification, but the dimension of the equalizer $v$ associated with CC-IFC algorithm is $P L N_{1}$ which is $N_{1}$ times that $(P L)$ associated with Algorithm 1 (see (28)). On the other hand, the computational load of the user identification in (S1) and signal recovery in (S2) of Algorithm 1 is much smaller than that of the source extraction using FKMA which, as mentioned in Section 3.1, is significantly much faster than gradient-type algorithms [6-8]. Consequently, Algorithm 1 is also computationally faster than the CC-IFC algorithm.

\section{MULTIRATE BMDA USING INSTANTANEOUS MIMO SIGNAL MODEL}

The proposed multirate BMDA using the instantaneous MIMO signal model $y[n]$ given by (24) comprises estimation of all the source signals $u_{11}^{(l)}[n], l=1,2, \ldots, N_{1}$, including user identification, and recovery of the desired user's symbol sequence $u_{11}[n]$ from the obtained estimates $\hat{u}_{11}^{(l)}[n]$ 's. Some general assumptions for the instantaneous MIMO signal model $\mathrm{y}[n]$ given by (24) are as follows.

(A1) $u_{i j}[n]$ for all $i$ and $j$ are i.i.d. zero-mean non-Gaussian with $C_{4}\left\{u_{i j}[n]\right\} \neq 0$, and statistically independent of $u_{l q}[n]$ for all $(i, j) \neq(l, q)$ (i.e., the same assumption as (A1)).

(A2) The unknown $\mathscr{H}$ (which is a $(P+q) \times(\mathcal{K}+2 K)$ channel matrix for the VPG system, or a $(P+q) \times(3 \mathcal{K})$ channel matrix for the MC system) is of full column rank with $(P+q) \geq(\mathcal{K}+2 K)$ for the VPG system or with $(P+$ $q) \geq(3 \mathcal{K})$ for the MC system. 
(A3) $\mathrm{w}[n]$ is zero-mean Gaussian, and statistically independent of $\mathrm{u}[n]$.

As mentioned in (R2), not all the source signals in $\mathrm{u}[n]$ are mutually independent sources (random processes) because $\mathrm{u}[n]$ is correlated with $\mathrm{u}[n-1]$. Nevertheless, assumption (A1) implies the following fact.

Fact 2. $\mathrm{u}[n]$ for each fixed $n$ (see (26)) is a zero-mean nonGaussian random vector with all the random components being mutually statistically independent.

\subsection{Extraction of source signals}

Our goal is to design a $(P+q) \times 1$ linear combiner v such that its output

$$
\varepsilon[n]=\mathrm{v}^{T} \mathrm{y}[n]
$$

approximates one of the $N_{1}$ subsequences $u_{11}^{(l)}[n], l=1,2$, $\ldots, N_{1}$ (see (3)). It is also known [30] that under the assumption $(\mathscr{A} 2)$, the noise-free assumption, and Fact 2 , the optimum $\varepsilon[n]$ by maximizing $J(\varepsilon[n]$ ) (see (30)) is exactly one source signal in $\mathrm{u}[n]$ except for an unknown scale factor. Therefore, for finite SNR, the iterative FKMA with $v=\mathrm{v}$ and $\psi[n]=\mathrm{y}[n]$ (presented in Section 3.1) can be applied to obtain one source estimate

$$
\varepsilon[n] \simeq \alpha_{i j, m}^{(l)} u_{i j, m}^{(l)}[n],
$$

where the subscripts $i, j, m$ and the superscript $l$ are unknown, and $\alpha_{i j, m}^{(l)}$ is an unknown scale factor.

As mentioned in (R4), a well-designed initial condition for $\mathbf{v}[n]$ associated with the convolutional model $\mathbf{y}[n]$ reported in [8] is preferred for efficient extraction of the desired source signals (virtual users) $u_{11}^{(\ell)}[n]$ 's. Again, a welldesigned initial condition for $\mathrm{v}$ (which, however, is never a special case of the initial condition for $\mathbf{v}[n]$ reported in [8]) is also needed so that $\varepsilon[n] \simeq \alpha_{11,0}^{(\ell)} u_{11,0}^{(\ell)}[n]=\alpha_{11}^{(\ell)} u_{11}^{(\ell)}[n]$ for some $\ell$. Next, let us present how to find a good initial condition for $\mathrm{v}$.

Let

$$
\mathrm{v}_{\mathrm{t}}=\left(\mathbf{0}_{\mathrm{t}}^{T},\left(\mathbf{c}_{11}^{(\ell)}\right)^{T}, \mathbf{0}_{(q-\mathrm{t})}^{T}\right)^{T},
$$

where $\mathbf{c}_{11}^{(\ell)}$ is given by (17) and

$$
\tau=\arg \max _{\mathrm{t}}\left\{J\left(\mathrm{v}_{\mathrm{t}}\right), \mathrm{t}=0,1, \ldots, q-1\right\} .
$$

Then, an initial condition $\mathrm{v}^{(0)}$ is suggested as follows:

$$
\mathrm{v}^{(0)}=\mathrm{v}_{\tau} .
$$

With the suggested initial condition $\mathrm{v}^{(0)}$ and (24) substituted into (46), one can obtain

$$
\begin{aligned}
\mathcal{E}[n] & =\mathrm{v}_{\tau}^{T} \mathrm{y}[n]=\sum_{i=1}^{G} \sum_{j=1}^{K_{i}} \sum_{l=1}^{N_{i}} \sum_{m=-1}^{1} \eta_{i j, m}^{(l)} u_{i j, m}^{(l)}[n]+w[n] \\
& =\eta_{11,0}^{(\ell)} u_{11,0}^{(\ell)}[n]+\mathrm{ISI}+\mathrm{MAI}+w[n],
\end{aligned}
$$

where $w[n]=\mathrm{v}_{\tau}^{T} \mathrm{w}[n], \eta_{i j, m}^{(l)}=\mathrm{v}_{\tau}^{T} \mathrm{~h}_{i j, m}^{(l)}$ and

$$
\left|\eta_{11,0}^{(\ell)}\right| \simeq \beta_{1}\left|g_{11}[\tau]\right| \gg\left|\eta_{i j, m}^{(l)}\right|, \quad \forall(i, j, m, l) \neq(1,1,0, \ell),
$$

where $\beta_{1}=P_{1}$ for the VPG system and $\beta_{1}=P$ for the MC system, and we have used (18) and (19) in the derivation of (52). From (51) and (52), one can infer that $g_{11}[\tau]$ is basically the strongest path in $g_{11}[k]$ and the ISI and MAI in $\varepsilon[n]$ have been substantially suppressed, thus efficiently leading to the optimum $\varepsilon[n]=\hat{u}_{11,0}^{(\ell)}[n]=\hat{u}_{11}^{(\ell)}[n]$. However, even with the use of the above initial condition $\mathrm{v}_{\tau}$, it cannot be guaranteed that $(i, j, m, l)=(1,1,0, \ell)$, and therefore $(i, j, m, l)$ needs to be identified as well.

Assume that all the users' spreading sequences are known in advance. Now let us present how to identify $(i, j, m, l)$ from $\varepsilon[n]$ given by $(47)$ and the following channel estimate $\hat{\mathrm{h}}_{i j, m}^{(l)}$ $[6,7,26,30]$ :

$$
\begin{aligned}
\hat{\mathrm{h}}_{i j, m}^{(l)} & =\frac{E\left\{\mathrm{y}[n] \varepsilon^{*}[n]\right\}}{E\left\{|\varepsilon[n]|^{2}\right\}} \quad(\text { by }(24) \text { and }(47)) \\
& \simeq \frac{\mathrm{h}_{i j, m}^{(l)}}{\alpha_{i j, m}^{(l)}}=\frac{1}{\alpha_{i j, m}^{(l)}} \mathrm{C}_{i j, m}^{(l)} \mathbf{g}_{i j} \quad(\text { by }(19)) .
\end{aligned}
$$

Let $\mathbf{b}=\left(b_{1}, b_{2}, \ldots, b_{p}\right)^{T}$ be a $p \times 1$ vector and

$$
\Lambda(\mathbf{b})=\Lambda(\beta \mathbf{b})=\frac{\sum_{i=1}^{p}\left|b_{i}\right|^{4}}{\left(\sum_{i=1}^{p}\left|b_{i}\right|^{2}\right)^{2}}, \quad \forall \beta \neq 0
$$

which is also an "entropy measure" of a finite sequence $b_{i}$ and $0 \leq \Lambda(\mathbf{b}) \leq 1$ (with minimum entropy corresponding to $\Lambda(\mathbf{b})=1)[6-8]$. Let

$$
\mathbf{a}_{\mathrm{ij}, \mathrm{m}}^{(\mathrm{l})}=\left(\mathbf{C}_{\mathrm{ij}, \mathrm{m}}^{(\mathrm{l})}\right)^{H} \cdot \hat{\mathrm{h}}_{i j, m}^{(l)}, \quad \forall \mathrm{i}, \mathrm{j}, \mathrm{m}, \mathrm{l} .
$$

By the fact that $c_{i j}[k]$ is a pseudonoise sequence and by (18), (19), (20), (54), and (56), one can easily prove that as $\mathrm{h}_{i j, m}^{(l)} \neq$ $\mathbf{0}_{P+q}$,

$$
\mathbf{a}_{\mathrm{ij}, \mathrm{m}}^{(\mathrm{l})} \simeq \begin{cases}\frac{\beta_{i} \cdot \mathbf{g}_{i j}}{\alpha_{i j, 0}^{(l)}}, & (\mathrm{i}, \mathrm{j}, \mathrm{m}, \mathrm{l})=(i, j, 0, l), \\ \frac{\operatorname{diag}\{q, q-1, \ldots, 1,0\} \cdot \mathbf{g}_{i j}}{\alpha_{i j,-1}^{(l)},}, & (\mathrm{i}, \mathrm{j}, \mathrm{m}, \mathrm{l})=(i, j,-1, l), \\ \frac{\operatorname{diag}\{0,1, \ldots, q-1, q\} \cdot \mathbf{g}_{i j}}{\alpha_{i j, 1}^{(l)}}, & (\mathrm{i}, \mathrm{j}, \mathrm{m}, \mathrm{l})=(i, j, 1, l),\end{cases}
$$

where $\beta_{i}=P_{i}$ for the VPG system and $\beta_{i}=P$ for the MC system, and that as $\mathrm{h}_{i j, m}^{(l)} \neq \mathbf{0}_{P+q}, \mathbf{a}_{\mathrm{ij}, \mathrm{m}}^{(\mathrm{l})}$ appears as a finite random sequence for $(\mathrm{i}, \mathrm{j}, \mathrm{m}, \mathrm{l}) \neq(i, j, m, l)$, implying

$$
\Lambda\left(\mathbf{a}_{i j, m}^{(l)}\right) \simeq \Lambda\left(\mathbf{g}_{i j}\right) \gg \Lambda\left(\mathbf{a}_{\mathrm{ij}, \mathrm{m}}^{(\mathrm{l})}\right), \quad \forall(\mathrm{i}, \mathrm{j}, \mathrm{m}, \mathrm{l}) \neq(i, j, m, l) .
$$

Then, the proposed UIA for identifying the $(i, j, m, l)$ associated with the $\varepsilon[n]=\hat{u}_{i j, m}^{(l)}[n]$ is as follows. 
(U1) Compute $\Lambda\left(\mathbf{a}_{\mathrm{ij}, \mathrm{m}}^{(\mathrm{l})}\right)$ for all $\mathrm{i}, \mathrm{j}, \mathrm{m}, \mathrm{l}$ using (53), (55), and (56).

(U2) Identify $(i, j, m, l)$ by

$$
(\hat{i}, \hat{j}, \hat{m}, \hat{l})=\arg \max _{(\mathrm{i}, \mathrm{j}, \mathrm{m}, \mathrm{l})}\left\{\Lambda\left(\mathbf{a}_{\mathrm{ij}, \mathrm{m}}^{(\mathrm{l})}\right)\right\} .
$$

If $(\hat{i}, \hat{j}, \hat{m}, \hat{l})=(1,1,0, \ell)$, then the desired source estimate $\varepsilon[n]=\hat{u}_{11,0}^{(\ell)}[n]=\hat{u}_{11}^{(\ell)}[n]$ is obtained, otherwise, one has to update $\mathrm{y}[n]$ by $\mathrm{y}[n]-\widehat{\mathrm{h}}_{i j, m}^{(l)} \varepsilon[n]$ (cancellation or deflation processing) and then repeat the preceding signal processing stage (source extraction followed by user identification) until an estimate $\hat{u}_{11}^{(\ell)}[n]$ is obtained (except for a scale factor).

\subsection{Recovery of the desired user's symbol sequence}

To obtain $\hat{u}_{11}[n]$ from all the desired virtual users' symbol estimates $\hat{u}_{11}^{(l)}[n], l=1,2, \ldots, N_{1}$, requires only the estimation of relative scale factors $\lambda_{11}^{(l)}, l=1,2, \ldots, N_{1}$, given by (44). However, a chip-rate channel estimate $\hat{g}_{11}^{(l)}[k]$ can be easily obtained via

$$
\begin{aligned}
\hat{\mathbf{g}}_{11}^{(l)} & =\left(\hat{g}_{11}^{(l)}[0], \hat{g}_{11}^{(l)}[1], \ldots, \hat{g}_{11}^{(l)}[q]\right)^{T}=\left(\mathbf{C}_{11,0}^{(l)}\right)^{H} \hat{\mathrm{h}}_{11,0}^{(l)} \\
& \simeq \frac{\beta_{1} \cdot \mathbf{g}_{11}}{\alpha_{11}^{(l)}}(\text { by }(20) \text { and }(54)),
\end{aligned}
$$

where the approximation $\left(\mathbf{C}_{11,0}^{(l)}\right)^{H} \mathbf{C}_{11,0}^{(l)} \simeq \beta_{1} \mathbf{I}$ (identity matrix) has been used in the derivation of (60) since $c_{i j}^{(l)}[k]$ is a pseudonoise sequence. Therefore, the parameter $\lambda_{11}^{(l)}$ can then be estimated by (43), (44), and (60). Finally, by (3), (44), and (47), we obtain the symbol sequence estimate of the desired user as follows:

$$
\widehat{u}_{11}[n]=\lambda_{11}^{(l)} \cdot \hat{u}_{11}^{(l)}[\tilde{n}] \simeq \alpha_{11}^{(1)} u_{11}[n],
$$

where $l=\left(n\right.$ modulo $\left.N_{1}\right)+1$ and $\tilde{n}=(n-l+1) / N_{1}$, and $\alpha_{11}^{(1)}$ is the unknown scale factor in the estimate $\hat{u}_{11}[n]$.

Let us summarize the resultant multirate BMDA using the instantaneous signal model $\mathrm{y}[n]$, referred to as Algorithm 2, as follows.

Algorithm 2. (S1) As presented in Section 4.1, obtain $\hat{u}_{11}^{(l)}[n]$, $l=1,2, \ldots, N_{1}$, using the FKMA with $v=\mathrm{v}$ and $\psi[n]=$ $\mathrm{y}[n]$, and the proposed UIA.

(S2) As presented in Section 4.2, obtain $\hat{u}_{11}[n]$ from $\hat{u}_{11}^{(l)}[n], l=1,2, \ldots, N_{1}$, using (61).

Let us conclude this subsection about the proposed Algorithm 2 with the following remark.

(R7) Remark (R5) also applies to Algorithm 2 as long as the discrete-time signal vector $\mathrm{y}[n]$ given by $(24)$ is obtained.

\subsection{Performance and complexity comparison with Algorithm 1}

Prior to discussing the performance and complexity of the proposed Algorithms 1 and 2, let us briefly discuss the main assumptions made by the two algorithms. Assumptions (A1) (leading to Fact 1) and (A2) guarantee the perfect recovery of the desired users' symbol sequence for the case that $N=\infty$ and SNR $=\infty$ for Algorithm 1, so do assumptions (AA1) (leading to Fact 2 ) and (A2) for Algorithm 2. The condition on $P$ and $\mathcal{K}$, that is, $P \geq \mathcal{K}$, in assumption (A2) is the same for both the VPG system and MC system, while that in assumption $(\mathscr{A} 2)$ is $(P+q) \geq(\mathcal{K}+2 K)$ for the VPG system and $(P+q) \geq 3 \mathcal{K}$ for the MC system, implying that Algorithm 1 may allow more users than Algorithm 2 for an assigned value of $P$ (maximum spreading factor).

Now let us discuss the performance and complexity of Algorithms 1 and 2 by comparing their signal models (a convolutional MIMO model versus an instantaneous MIMO model) for the VPG system. Comparing the equalizer output $e[n]$ (see (27)) (the output of a linear equalizer $\mathbf{v}[n]$ of length $L)$ associated with Algorithm 1 and $\varepsilon[n]$ (see (46)) associated with Algorithm 2, one can easily see that the former is actually a special case of the latter if $\psi[n]$ (see (27) and (29)) is treated as the following instantaneous MIMO signal model:

$$
\begin{aligned}
\psi[n] & =\left(\mathbf{y}^{T}[n], \mathbf{y}^{T}[n-1], \ldots, \mathbf{y}^{T}[n-L+1]\right)^{T} \\
& =\widetilde{\mathscr{H}} \tilde{\mathrm{u}}[n]+\tilde{\mathrm{w}}[n],
\end{aligned}
$$

where

$$
\begin{aligned}
& \widetilde{\mathbf{u}}[n]=\left(\mathbf{u}^{T}[n], \mathbf{u}^{T}[n-1], \ldots,\right. \\
& \left.\mathbf{u}^{T}[n-L]\right)^{T}, \quad((L+1) \mathcal{K}) \times 1 \text { vector }, \\
& \tilde{\mathrm{w}}[n]=\left(\mathbf{w}^{T}[n], \mathbf{w}^{T}[n-1], \ldots,\right. \\
& \left.\mathbf{w}^{T}[n-L+1]\right)^{T}, \quad(P L) \times 1 \text { vector }, \\
& \widetilde{\mathscr{H}}=\left(\mathrm{H}_{1}^{T}, \mathrm{H}_{2}^{T}, \ldots, \mathrm{H}_{L}^{T}\right)^{T}, \quad(P L) \times((L+1) \mathcal{K}) \text { matrix }
\end{aligned}
$$

in which

$$
\begin{aligned}
& \mathrm{H}_{r}=\left(\mathbf{O}_{P \times(\mathcal{K} \cdot(r-1))}, \mathbf{H}[0], \mathbf{H}[1],\right. \\
&\left.\mathbf{O}_{P \times(\mathcal{K} \cdot(L-r))}\right), \quad P \times(L+1) \mathcal{K} \text { matrix },
\end{aligned}
$$

where $\mathbf{H}[n]$ is given by (12) and $\mathbf{O}_{p \times q}$ denotes a $p \times q$ zero matrix.

By (4), (8), and (13), it can be inferred that $\mathbf{H}[0]$ is basically of full column rank since $P \geq \mathcal{K}$, and $\mathbf{H}[1]=\left(\mathbf{O}_{P \times\left(N_{1}-1\right)}\right.$, $\left.\mathbf{h}_{11}^{\left(N_{1}\right)}[1], \mathbf{O}_{P \times\left(N_{1}-1\right)}, \mathbf{h}_{12}^{\left(N_{1}\right)}[1], \ldots, \mathbf{O}_{P \times\left(N_{G}-1\right)}, \mathbf{h}_{G K_{G}}^{\left(N_{G}\right)}[1]\right)$. Therefore, by removing zero column vectors in $\widetilde{\mathscr{H}}$ (due to zero column vectors in $\mathbf{H}[1])$, the channel matrix $\widetilde{\mathscr{H}}$ reduces to a (full column rank) $(P L) \times(L \mathcal{K}+K)$ matrix and $\tilde{u}[n]$ also reduces to an $(L \mathcal{K}+K) \times 1$ vector. Moreover, under assumption (A1), it can be easily seen that Fact 2 also applies to $\widetilde{\mathrm{u}}[n]$ (see $(63))$. Therefore, corresponding to $\varepsilon[n]$ given by (46), the extracted source $e[n]=v^{T} \psi[n]$ (see (27)) obtained by Algorithm 1 turns out to be an estimate of one source component in $\tilde{\mathrm{u}}[n]$. In other words, $e[n]$ given by (36) is true since $u_{i j}^{(l)}\left[n-\tau_{i j}^{(l)}\right]$ is exactly a component of $\tilde{\mathrm{u}}[n]$ (i.e., a source in $\tilde{\mathrm{u}}[n])$ by (10) and (63).

The above analysis also shows that Algorithms 1 and 2 are theoretically consistent in estimating the desired symbol sequence $u_{11}[n]$. However, in practical applications where both 
the data length and SNR are finite, their performance and/or complexity may be very different as discussed next.

Because the dimensions of the equalizer vector $v$ (see (28)) for Algorithm 1 and $\mathrm{v}$ (see (46)) for Algorithm 2 are $\operatorname{dim}(\nu)=P L$ and $\operatorname{dim}(\mathrm{v})=P+q$, respectively, one can see that $\operatorname{dim}(\mathrm{v}) \ll \operatorname{dim}(\nu)$ if $L>1$, implying that Algorithm 1 may significantly overparameterize the equalizer for finite data length and finite SNR (leading to larger estimation errors), although Algorithms 1 and 2 have the same performance (perfect estimation of the desired symbol sequence) for infinite data length and infinite SNR. On the other hand, one can observe, from (18), (24), (25), and (26), that the channel column associated with each desired virtual user's symbol sequence $u_{11,0}^{(l)}[n]$ in the instantaneous MIMO signal model $\mathrm{y}[n]$ for Algorithm 2 is $\mathrm{h}_{11,0}^{(l)}=$ $\left(h_{11}^{(l)}[0], h_{11}^{(l)}[1], \ldots, h_{11}^{(l)}[P+q-1]\right)^{T}$ which includes all the channel paths (i.e., full diversity), in spite of much smaller dimension of the instantaneous MIMO signal model $\mathscr{H}$ (see (25)) associated with Algorithm 2 than that of $\widetilde{\mathscr{H}}$ (see (65)) associated with Algorithm 1. So we can conclude that Algorithm 2 outperforms Algorithm 1 for finite data length and finite SNR, and meanwhile the computational complexity of the former is also much lower than that of the latter thanks to $\operatorname{dim}(\mathrm{v}) \ll \operatorname{dim}(\nu)$.

The above performance and complexity comparison of Algorithms 1 and 2 can be similarly conducted for the MC system, and the same conclusion can be obtained.

\section{MULTIPLE RECEIVE ANTENNAS}

In this section, we extend Algorithms 1 and 2 presented in Sections 3 and 4 (where one receive antenna was considered) to multiple receive antennas. Assume that the receiver is equipped with $Q$ antennas and that $u[n]$ is the symbol sequence of the desired user. Two approaches for the extension are considered. One is full-dimension (joint) space-time processing and the other is temporal processing followed by blind maximum ratio combining (BMRC) $[6-8,26]$ for the estimation of $u[n]$.

\subsection{Full-dimension space-time processing}

Let $\mathbf{y}_{r}[n], \mathrm{y}_{r}[n], \mathbf{H}_{r}[n]$, and $\mathscr{H}_{r}$ denote the $P \times 1$ signal vector $\mathrm{y}[n]($ see $(9)),(P+q) \times 1$ signal vector $\mathrm{y}[n]($ see $(24)), P \times \mathcal{K}$ convolutional MIMO channel $\mathbf{H}[n]$ (see (12)), and $P \times 3 \mathcal{K}$ instantaneous MIMO channel $\mathscr{H}$ (see (25)), respectively, at the $r$ th receive antenna. By concatenating $\mathbf{y}_{r}[n], r=1,2, \ldots$, $Q$, and $\mathrm{y}_{r}[n], r=1,2, \ldots, Q$, one can obtain a $(P Q) \times 1$ convolutional signal model

$$
\overline{\mathbf{y}}[n]=\left(\mathbf{y}_{1}^{T}[n], \mathbf{y}_{2}^{T}[n], \ldots, \mathbf{y}_{Q}^{T}[n]\right)^{T}=\overline{\mathbf{H}}[n] * \mathbf{u}[n]+\overline{\mathbf{w}}[n]
$$

and $\mathrm{a}((P+q) Q) \times 1$ instantaneous signal model

$$
\overline{\mathrm{y}}[n]=\left(\mathrm{y}_{1}^{T}[n], \mathrm{y}_{2}^{T}[n], \ldots, \mathrm{y}_{Q}^{T}[n]\right)^{T}=\overline{\mathscr{H}} \mathrm{u}[n]+\overline{\mathrm{w}}[n]
$$

where $\overline{\mathbf{H}}[n]=\left(\mathbf{H}_{1}^{T}[n], \mathbf{H}_{2}^{T}[n], \ldots, \mathbf{H}_{Q}^{T}[n]\right)^{T}, \overline{\mathscr{H}}=\left(\boldsymbol{H}_{1}^{T}, \mathscr{H}_{2}^{T}\right.$, $\left.\ldots, \mathscr{H}_{Q}^{T}\right)^{T}$, and $\overline{\mathbf{w}}[n]$ and $\overline{\mathrm{w}}[n]$ are a $(P Q) \times 1$ noise vector and a $((P+q) Q) \times 1$ noise vector, respectively.

It is straightforward to apply Algorithm 1 presented in Section 3 to process $\overline{\mathbf{y}}[n]$ and Algorithm 2 presented in Section 4 to process $\bar{y}[n]$ to estimate $u[n]$ (with space diversity gain).

\subsection{Temporal processing followed by $B M R C$}

Let $\hat{u}_{r}[n]$ be the obtained estimate of $u[n]$ by processing the received signal at the $r$ th antenna using either Algorithm 1 or Algorithm 2. Therefore, $\hat{u}_{r}[n]$ can be expressed as

$$
\hat{u}_{r}[n] \simeq \begin{cases}\alpha_{r} u\left[n-\tau_{r}\right], & \text { for Algorithm 1, } \\ \alpha_{r} u[n], & \text { for Algorithm 2, }\end{cases}
$$

where $\alpha_{r}$ and $\tau_{r}$ are the unknown scale factor and time delay, respectively, associated with the $r$ th antenna. The relative time delays $\tau_{r}-\tau_{1}$ can be easily estimated by cross-correlation of $\hat{u}_{r}[n]$ and $\hat{u}_{1}[n][6,7]$. Assume that $\tau_{1}=0$ for simplicity. After the time delay compensation, $\hat{u}_{r}[n]$ can be modeled as follows:

$$
\widehat{u}_{r}[n]=\alpha_{r} u[n]+\omega_{r}[n], \quad r=1,2, \ldots, Q,
$$

(an instantaneous single-input multiple-output system) where $\omega_{r}[n]$ is the estimation error associated with the $r$ th antenna.

Let $v$ be a $Q \times 1$ linear combiner and $\psi[n]=$ $\left(\hat{u}_{1}[n], \hat{u}_{2}[n], \ldots, \hat{u}_{Q}[n]\right)^{T}$. By using the BMRC algorithm (which also uses FKMA) reported in $[6-8,26]$, the obtained estimate

$$
\widehat{u}[n]=v^{T} \psi[n] \simeq \alpha u[n]
$$

can be shown to have the same signal-to-interference-plusnoise ratio (SINR) as the nonblind MMSE combiner (which requires $\alpha_{r}$ in (70) for all $r$ given in advance) provided that $\omega_{r}[n]$ is approximately zero-mean Gaussian for all $r$. Let us conclude this section with the following remark.

(R8) The estimate $\hat{u}[n]$ obtained by the full-dimension space-time processing approach is theoretically optimum without need of time delay compensation, while that obtained by the approach of temporal processing followed by BMRC is suboptimum. However, the former requires a search in a higher-dimensional space $(\operatorname{dim}(\bar{\nu})=P L Q$ for Algorithm 1 and $\operatorname{dim}(\overline{\mathrm{v}})=(P+q) Q$ for Algorithm 2), and thus leading to higher computational complexity on one hand and possibly larger estimation errors on the other hand for finite data length and finite SNR.

\section{SIMULATION RESULTS}

This section presents some simulation results to justify the effectiveness of the proposed two multirate BMDAs, Algorithm 1 and Algorithm 2, together with a performance comparison with two nonblind MMSE detectors (one associated with the convolutional MIMO signal model $\mathbf{y}[n]$ 
given by (9) and the other associated with the instantaneous MIMO signal model $\mathbf{y}[n]$ given by (24)), Ma and Tugnait's blind CC-IFC algorithm [13], and the blind MV receiver proposed by Tsatsanis et al. [11] and Tsatsanis and $\mathrm{Xu}$ [19]. Note that Algorithm 1 and the blind CC-IFC algorithm are based on the convolutional MIMO signal model $\mathbf{y}[n]$, whereas Algorithm 2 and the blind MV receiver are based on the instantaneous MIMO signal model y $[n]$.

Consider a six-user asynchronous dual rate DS/CDMA system with $K_{1}=K_{2}=3$ and $R_{1}=2 R_{2}$. For the VPG system, the spreading sequences for group 1 are Gold codes with $P_{1}=$ 31 while those for group 2 with $P_{2}=62$ are formed by two Gold codes of length equal to 31. For the MC system, all the $c_{i j}^{(l)}[k]$ 's (with the spreading factor $P=62$ ) are also formed by two Gold codes of length 31 . The synthetic signals $\mathbf{y}[n]$ and $y[n]$ were generated by (9) and (24), respectively, with $u_{i j}[n]$ being a binary sequence of \pm 1 (i.i.d. non-Gaussian sequence with $C_{4}\left\{u_{i j}[n]\right\}=-2$ ) and a 3-path channel for each user with $q=\max _{i j}\left\{d_{i j}\right\}=10$, the noise $w(t)$ given by (1) being a zero-mean Gaussian with $E\left\{|w(t)|^{2}\right\}=\sigma_{w}^{2}$. Then the synthetic signal y $n]$ was processed by the associated nonblind MMSE detector, Algorithm 2, and the blind MV receiver, and the synthetic signal $\mathbf{y}[n]$ was processed by the associated nonblind MMSE detector, Algorithm 1, and the blind CC-IFC algorithm with the length $L=3$ for the $P \times 1$ FIR equalizer $\mathbf{v}[n]$.

Fifty independent runs were performed for each simulation result for different values of the desired user's SNR, called input SNR, defined as

$$
\text { Input SNR }=\frac{E\left\{\left|y_{11}(t)\right|^{2}\right\}}{\sigma_{w}^{2}} .
$$

The output SINR $[6,8]$ of the desired user is used for the performance evaluation of the algorithms under test for near-far ratio $\left(\mathrm{NFR}=\mathscr{E} / E\left\{\left|y_{11}(t)\right|^{2}\right\}\right)$ equal to $0 \mathrm{~dB}$ and $10 \mathrm{~dB}$, where $\mathcal{E}=E\left\{\left|y_{i j}(t)\right|^{2}\right\}$ for $(i, j) \neq(1,1)$, that is, all the other users' powers are the same.

Figures 1 (a) (for NFR $=0 \mathrm{~dB}$ ) and 1 (b) (for NFR = $10 \mathrm{~dB}$ ) show the simulation results (output SINR versus the desired user's SNR (input SNR) for low-rate data length $N=2500)$ for the VPG system with one receive antenna employed. The corresponding results for the MC system are shown in Figures $1(\mathrm{c})$ and $1(\mathrm{~d})$. One can see, from Figures $1(\mathrm{a})$ and 1(b), that the performances of Algorithm 2 $(\bigcirc)$, Ma and Tuanait's CC-IFC algorithm $(\triangle)$, and the nonblind MMSE detector associated with $\mathrm{y}[n]$ (dashed line) are close to the performance of the nonblind MMSE detector associated with $\mathbf{y}[n]$ (solid line), and slightly superior to that of Algorithm $1(\diamond)$, and much better than that of the MV receiver $(\square)$ for the VPG system. The same conclusion applies to Figures 1(c) and 1(d) (the MC system) except that Algorithm 1 performs much better than the MV receiver, but much worse than the nonblind MMSE detectors, Algorithm 2, and the CC-IFC algorithm for NFR $=10 \mathrm{~dB}$.

Let us also show the results (corresponding to those shown in Figure 1 obtained through 50 independent runs) of bit error rate (BER) in Figure 2 that were obtained through 500 independent runs instead. One can see, from Figures 1 and 2, that all the relative performances between the algorithms under test are consistent. However, BERs are equal to zero in quite many cases (high SNR) and thus cannot be shown in Figure 2 due to insufficient independent runs. Therefore, output SINR is preferred to BER as the performance index of the algorithms under test with sufficient (but limited) simulation results.

On the other hand, Figures 3(a) and 3(b) show some results (output SINR versus low-rate data length $N$ for input $\mathrm{SNR}=10 \mathrm{~dB}$ ) for $\mathrm{NFR}=0 \mathrm{~dB}$ and $\mathrm{NFR}=10 \mathrm{~dB}$, respectively. One can observe, from Figures 3(a) and 3(b), that the performance of Algorithm 2 is slightly worse than that of the nonblind MMSE detectors, slightly superior to that of Algorithm 1 and the blind CC-IFC algorithm, and much better than that of the blind MV receiver. Note that their performance differences are larger for smaller $N$. The same conclusion applies to Figures 3(c) and 3(d) (the MC system) except that Algorithm 1, again, performs much better than the MV receiver, but much worse than the nonblind MMSE detectors, Algorithm 2, and the CC-IFC algorithm for $\mathrm{NFR}=10 \mathrm{~dB}$.

Figure 4 shows output SINR versus the desired user's SNR for NFR $=0 \mathrm{~dB}$ and NFR $=10 \mathrm{~dB}$, associated with the proposed Algorithm 1 (dashed line) and Algorithm 2 (solid line) using the approach of full-dimension space-time processing for multiple receive antennas. One can see, from Figure 4, that approximately a $3 \mathrm{~dB}$ and a $6 \mathrm{~dB}$ performance gain (antenna gain) are obtained by Algorithm 2 with 2 antennas $(\triangle)$ and 4 antennas $(\square)$, respectively, for both the VPG system and the MC system. On the other hand, one can see, from Figure 4, that approximately a $3 \mathrm{~dB}$ and a $5 \mathrm{~dB}$ performance gain are obtained by Algorithm 1 with 2 antennas and 4 antennas, respectively, for both the VPG system and the MC system except the case of NFR $=10 \mathrm{~dB}$ for the MC system (Figure $4(\mathrm{~d})$ ), where performance gains associated with Algorithm 1 decrease with SNR. On the other hand, Figures $4(\mathrm{a})-4$ (c) show that the performance of Algorithm 2 is uniformly superior to that of Algorithm 1, whereas Figure 4(d) shows that their difference becomes larger for $\mathrm{SNR} \geq 2 \mathrm{~dB}$ because performance gains of Algorithm 1 using multiple receive antennas become smaller for higher SNR.

All the results (corresponding to those shown in Figure 4) obtained using the approach of temporal processing followed by BMRC for multiple receive antennas are shown in Figure 5. Again, all the performance observations from Figure 4 basically apply to Figure 5 as well. On the other hand, one can see, from Figures 4 and 5, that the performance is basically the same for Algorithm 2 using either the approach of joint space-time processing or the approach of temporal processing followed by BMRC, whereas the performance for Algorithm 1 is somewhat better using the approach of temporal processing followed by BMRC than using the approach of joint space-time processing. These results are consistent with (R8).

The above simulation results demonstrate that the proposed Algorithm 2 performs nearly best for all the simulation cases (finite data length, finite SNR, and different NFRs) among all the blind algorithms under test. However, 


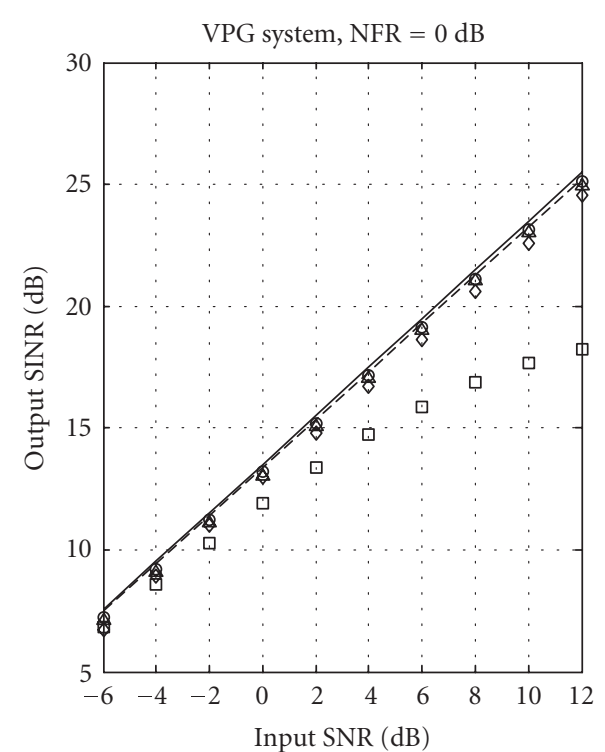

$\begin{array}{clll}- & \text { MMSE }(\mathrm{y}[n]) & \bigcirc & \text { Algorithm 2 } \\ --- & \text { MMSE }(\mathrm{y}[n]) & \triangle & \text { CC-IFC algorithm } \\ \diamond & \text { Algorithm 1 } & \square & \text { MV receiver }\end{array}$

(a) VPG system, NFR $=0 \mathrm{~dB}$

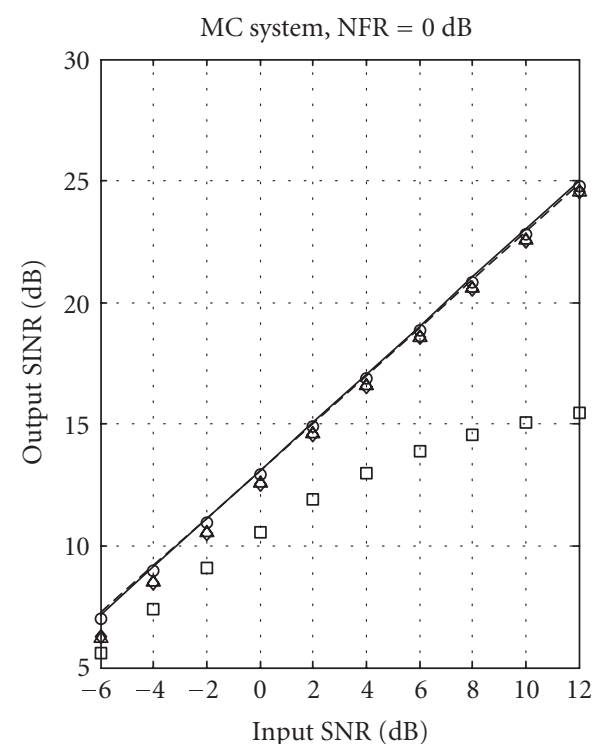

$\begin{array}{llll} & \text { MMSE }(\mathbf{y}[n]) & \bigcirc & \text { Algorithm 2 } \\ --- & \text { MMSE }(\mathrm{y}[n]) & \triangle & \text { CC-IFC algorithm } \\ \diamond & \text { Algorithm 1 } & \square & \text { MV receiver }\end{array}$

(c) MC system, NFR $=0 \mathrm{~dB}$

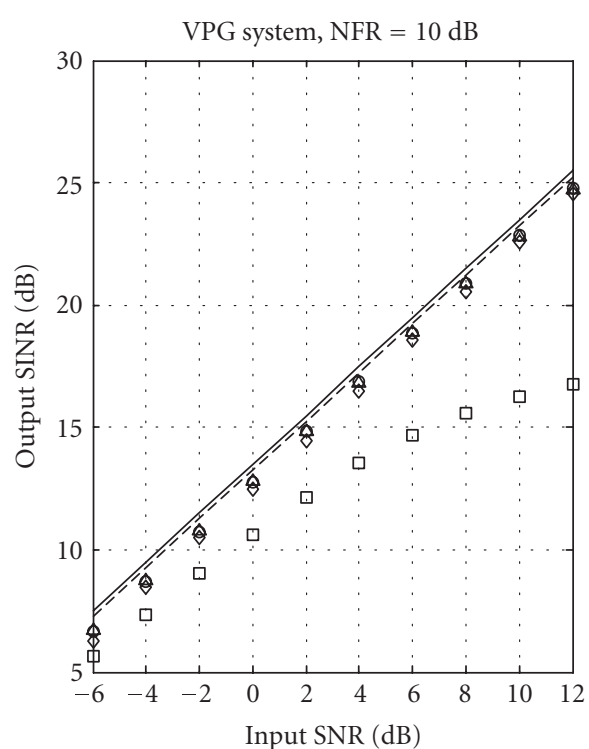

$\begin{array}{clll}- & \text { MMSE }(\mathbf{y}[n]) & \bigcirc & \text { Algorithm 2 } \\ --- & \text { MMSE }(\mathrm{y}[n]) & \triangle & \text { CC-IFC algorithm } \\ \diamond & \text { Algorithm 1 } & \square & \text { MV receiver }\end{array}$

(b) VPG system, NFR=10 dB.

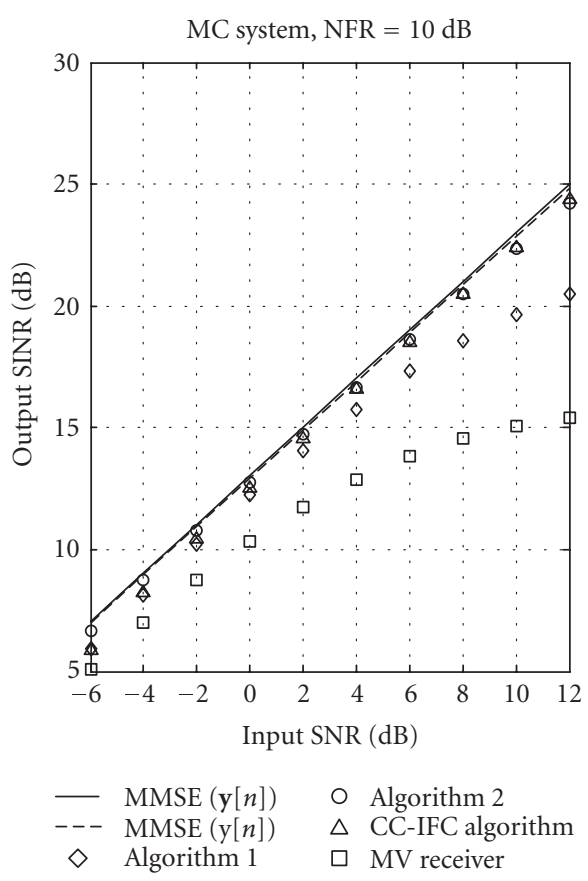

(d) MC system, NFR $=10 \mathrm{~dB}$

FIGURE 1: Simulation results (output SINR versus input SNR for low-rate data length $N=2500$ ) obtained by the nonblind MMSE detectors (associated with the convolutional model $\mathbf{y}[n]$ (solid line) and the instantaneous model $\mathrm{y}[n]$ (dashed line)), Algorithms $1(\diamond)$ and $2(\bigcirc)$, CC-IFC algorithm $(\triangle)$, and MV receiver $(\square)$ with one antenna used.

Algorithm 1 works well for all the simulation results except for the cases of the MC system for high NFR (= $10 \mathrm{~dB})$ because more successive cancellation stages were involved in source extraction, leading to performance degradation as stated in (R4). Nevertheless, unsuccessful extraction of the desired user's sequence for Algorithms 1 and 2 did not happen in the simulation, whereas it did happen to the CC-IFC algorithm in very few simulation results. On the other hand, 

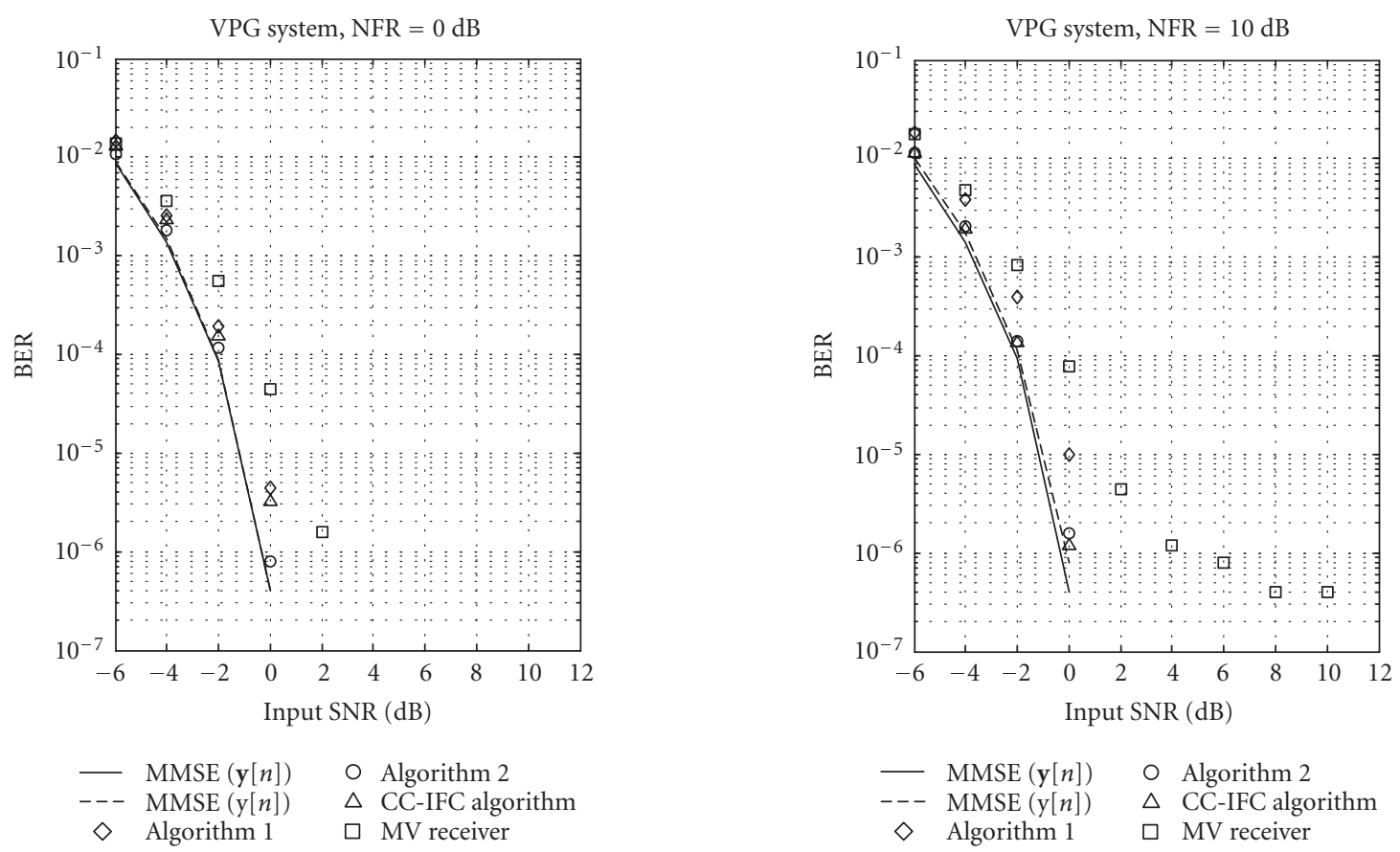

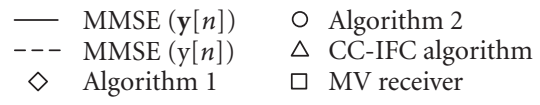

(a) VPG system, NFR $=0 \mathrm{~dB}$
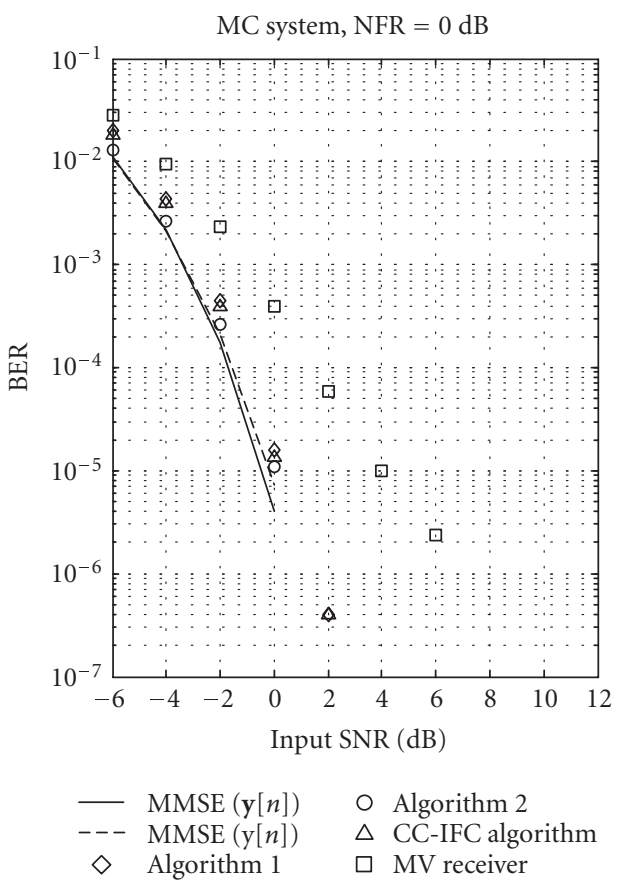

(c) MC system, NFR $=0 \mathrm{~dB}$ (b) VPG system, NFR=10 dB

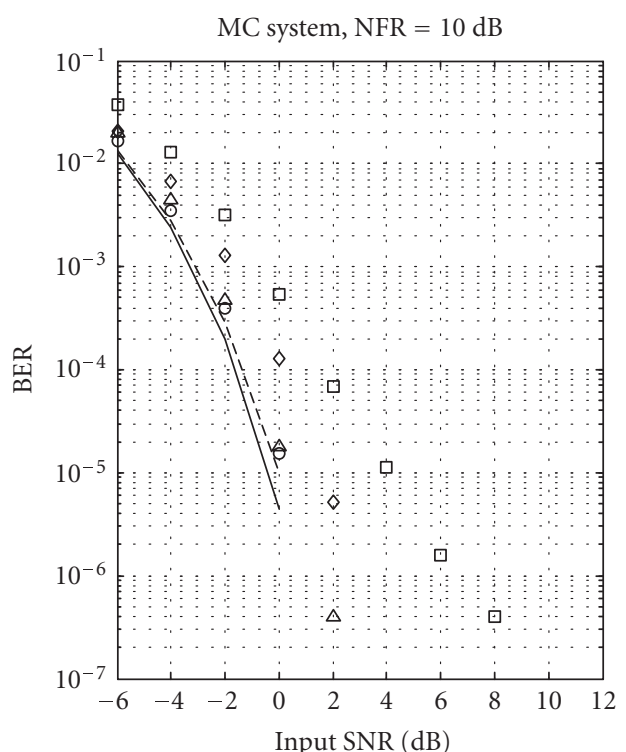

$\begin{array}{clll}- & \text { MMSE }(\mathbf{y}[n]) & \bigcirc & \text { Algorithm 2 } \\ --- & \text { MMSE }(\mathrm{y}[n]) & \triangle & \text { CC-IFC algorithm } \\ \diamond & \text { Algorithm 1 } & \square & \text { MV receiver }\end{array}$

(d) MC system, $\mathrm{NFR}=10 \mathrm{~dB}$

FIGURE 2: Simulation results (BER versus input SNR for low-rate data length $N=2500$ ) obtained by the nonblind MMSE detectors (associated with the convolutional model $\mathbf{y}[n]$ (solid line) and the instantaneous model $\mathrm{y}[n]$ (dashed line)), Algorithms $1(\diamond)$ and $2(\bigcirc)$, CC-IFC algorithm $(\triangle)$, and MV receiver $(\square)$ with one antenna used.

because $\operatorname{dim}(\mathrm{v})=P+q=62+10=72 \ll \operatorname{dim}(\nu)=P L=$ $62 \times 3=186$, the computational complexity of Algorithm 2 is much lower than that of Algorithm 1 as stated in Section 4.3, and thus much lower than the CC-IFC algorithm (see (R6)).
As a final remark, the performance of MMSE detector associated with the instantaneous MIMO signal model $\mathrm{y}[n]$ given by (24) is nearly the same as that associated with the convolutional MIMO signal model $\mathbf{y}[n]$ given by (9), implying the 


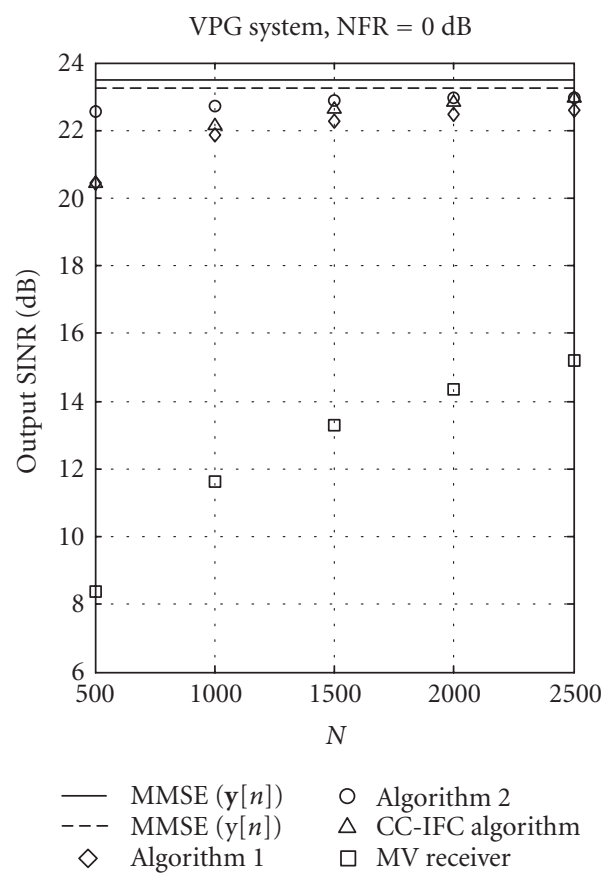

(a) VPG system, NFR $=0 \mathrm{~dB}$

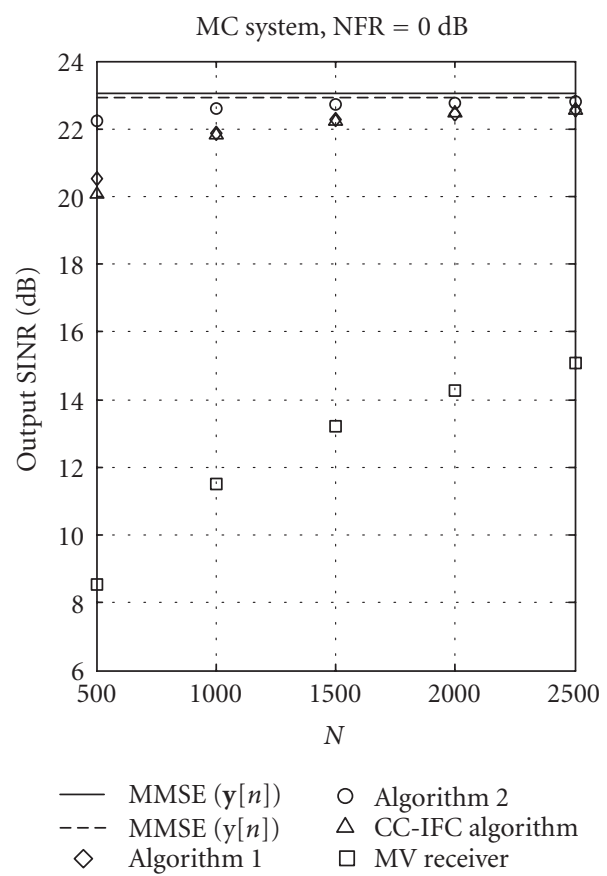

(c) MC system, NFR $=0 \mathrm{~dB}$

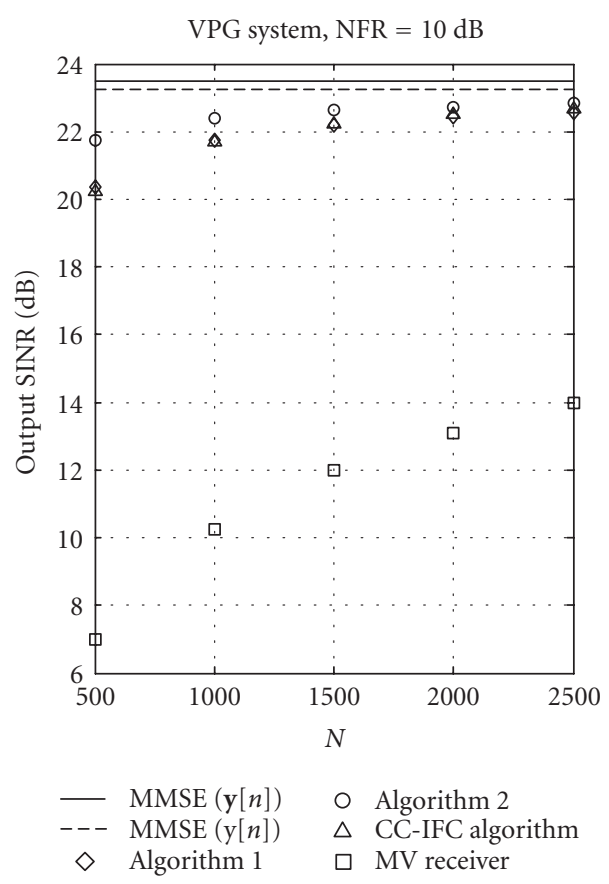

(b) VPG system, NFR=10 dB

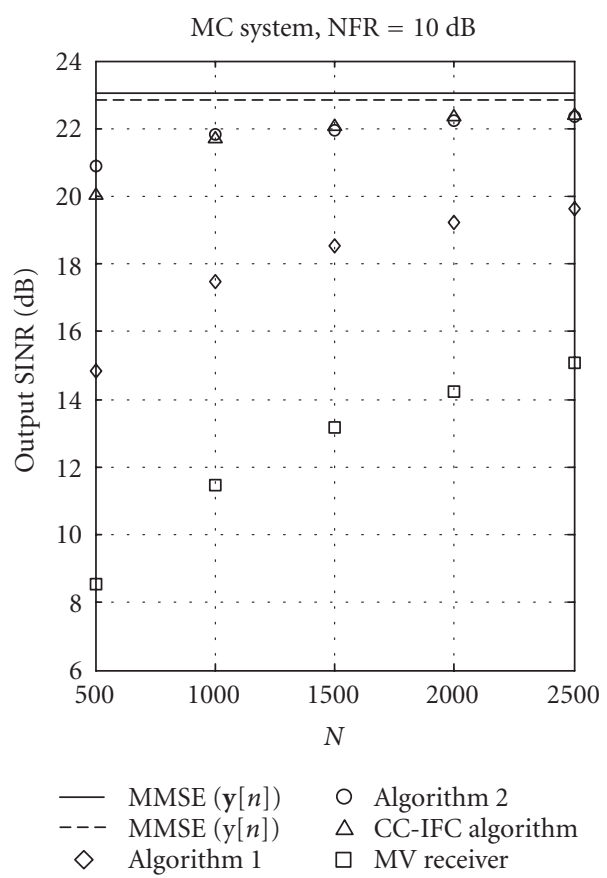

(d) MC system, NFR=10 dB

FIGURE 3: Simulation results (output SINR versus low-rate data length $N$ for input SNR $=10 \mathrm{~dB}$ ) obtained by the nonblind MMSE detectors (associated with the convolutional model $\mathbf{y}[n]$ (solid line) and the instantaneous model $y[n]$ (dashed line)), Algorithms $1(\diamond)$ and $2(\bigcirc)$, CC-IFC algorithm $(\triangle)$, and MV receiver $(\square)$ with one antenna used.

full diversity of the instantaneous MIMO signal model $\mathrm{y}[n]$ as stated in Section 4.3.

\section{CONCLUSIONS}

We have presented two multirate BMDAs for asynchronous multirate DS/CDMA systems (VPG and MC systems) equipped with a single or multiple receive antennas, Algorithm 1 and Algorithm 2, using the FKMA [6-8, 26], that therefore share the superexponential convergence rate and guaranteed convergence of the FKMA in source extraction. Some simulation results were provided to justify their effectiveness in addition to a performance comparison with 

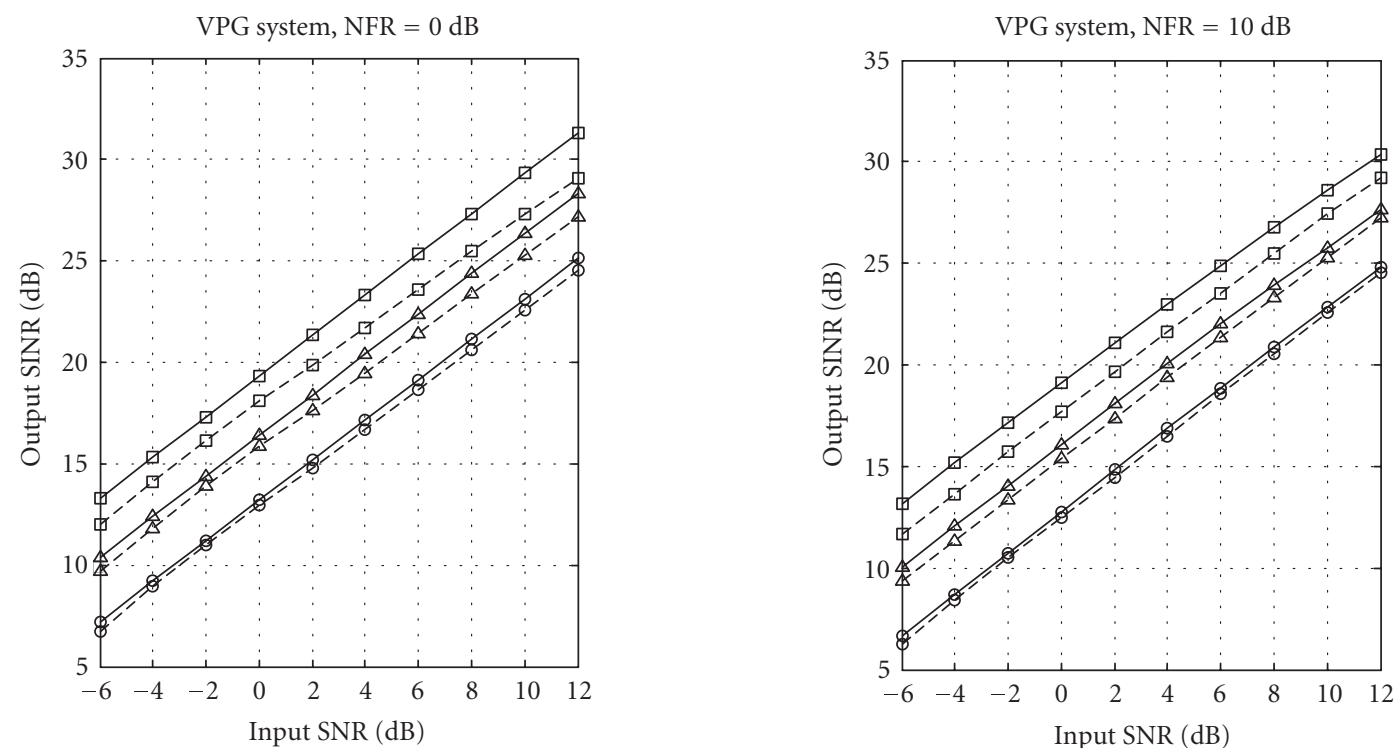

$$
\begin{array}{ll}
\text {--- Algorithm 1 } & \triangle Q=2 \\
\text { - Algorithm 2 } & \square Q=4 \\
Q \quad Q=1 &
\end{array}
$$

(a) VPG system, NFR $=0 \mathrm{~dB}$

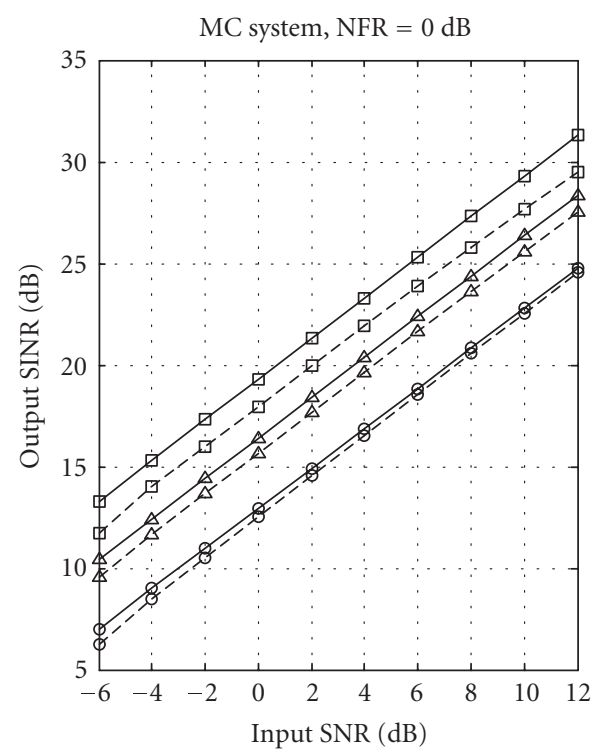

$$
\begin{array}{ll}
\text {--- Algorithm 1 } & \triangle Q=2 \\
\text { - Algorithm 2 } & \square Q=4 \\
\hline \quad Q=1 &
\end{array}
$$

(c) $\mathrm{MC}$ system, NFR $=0 \mathrm{~dB}$

$$
\begin{array}{ll}
\text {-- Algorithm 1 } & \triangle Q=2 \\
\text { - Algorithm 2 } & \square Q=4 \\
\hline Q=1 &
\end{array}
$$

(b) VPG system, NFR=10 dB

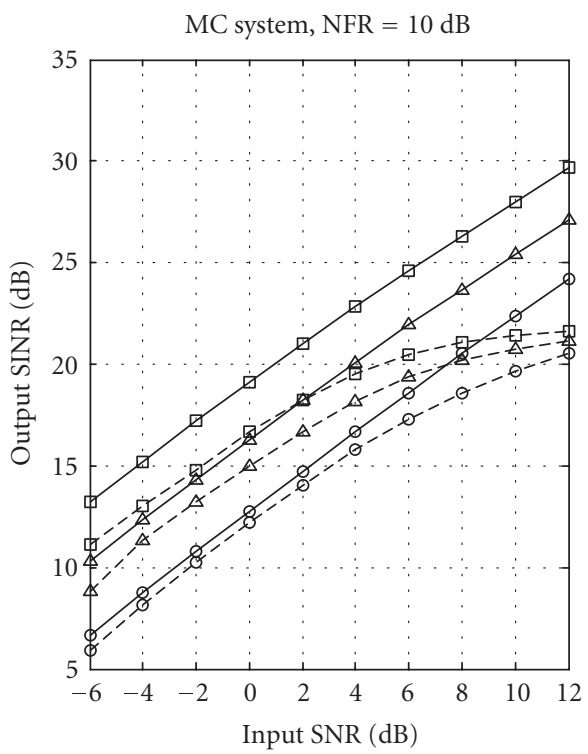

$$
\begin{array}{ll}
-- \text { Algorithm 1 } & \triangle Q=2 \\
\text { - Algorithm 2 } & \square Q=4 \\
Q=1 &
\end{array}
$$

(d) MC system, NFR=10 dB

FIgURE 4: Simulation results (output SINR versus input SNR for low-rate data length $N=2500$ ) obtained by Algorithms 1 (dashed line) and 2 (solid line) using the approach of full-dimension space-time processing with $1(\bigcirc), 2(\triangle)$, and $4(\square)$ antennas used.

the blind CC-IFC algorithm [13] and the blind MV receiver [11, 19], and to demonstrate that Algorithm 2 performs nearly best with performance close to the nonblind MMSE detector associated with the instantaneous MIMO signal model $\mathrm{y}[n]$ given by (24). Moreover, the computational complexity of Algorithm 2 is also much lower than that of Algorithm 1 and the blind CC-IFC algorithm. There- fore, Algorithm 2 can be a good candidate for practical applications in wireless communications.

\section{ACKNOWLEDGMENTS}

This work was supported by the National Science Council, ROC, under Grant NSC 94-2213-E-007-035. This work was 

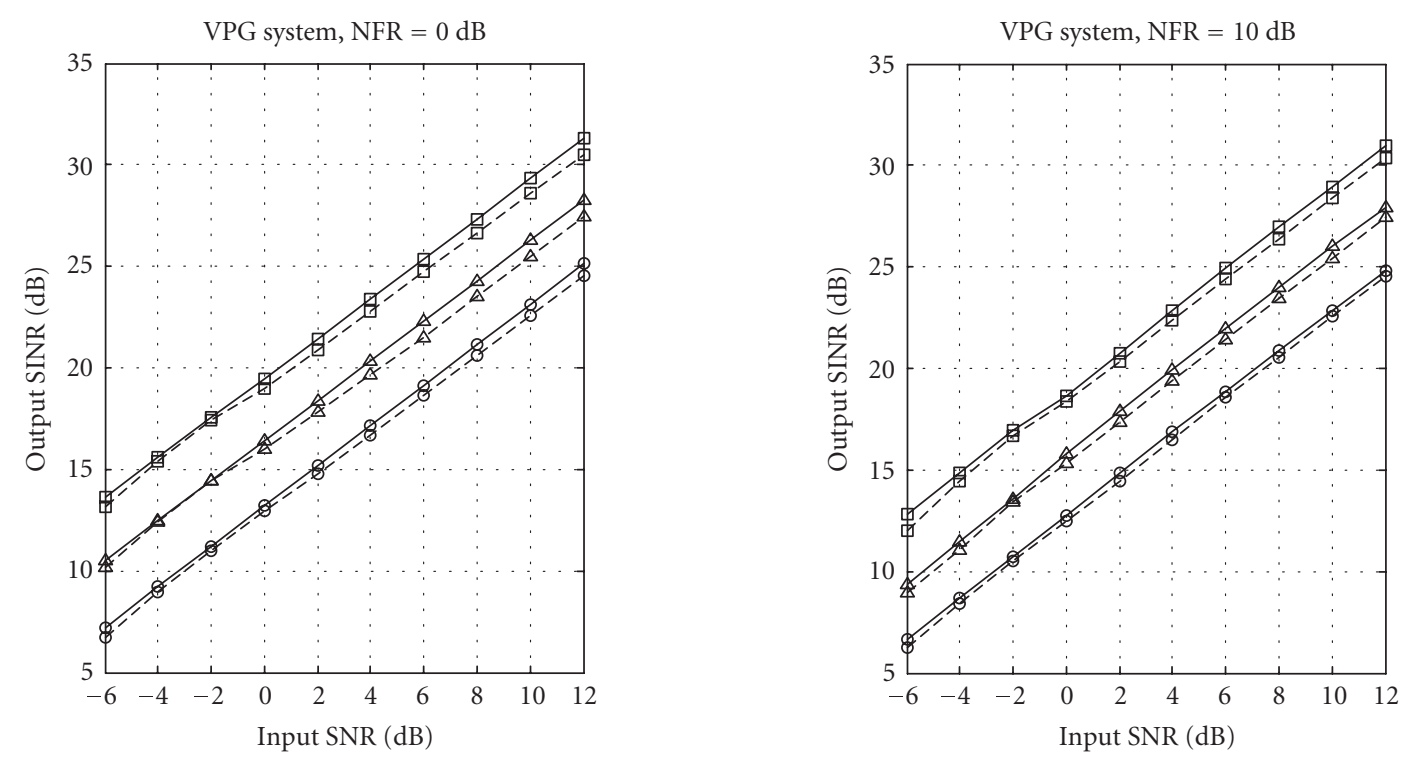

$$
\begin{array}{ll}
-- \text { Algorithm 1 } & \triangle Q=2 \\
- \text { Algorithm 2 } & \square Q=4 \\
\text { Q } Q=1 &
\end{array}
$$

(a) VPG system, NFR $=0 \mathrm{~dB}$

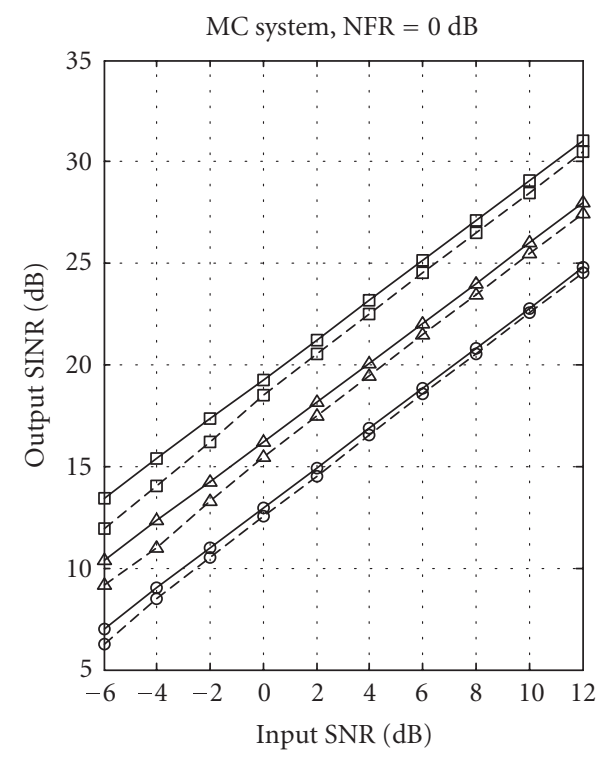

$$
\begin{array}{ll}
-- \text { Algorithm 1 } & \triangle Q=2 \\
- \text { Algorithm 2 } & \square Q=4 \\
& Q=1
\end{array}
$$

(c) MC system, NFR $=0 \mathrm{~dB}$

$$
\begin{array}{ll}
-- \text { Algorithm 1 } & \triangle Q=2 \\
- \text { Algorithm 2 } & \square Q=4 \\
\quad Q=1 &
\end{array}
$$

(b) VPG system, NFR=10 dB

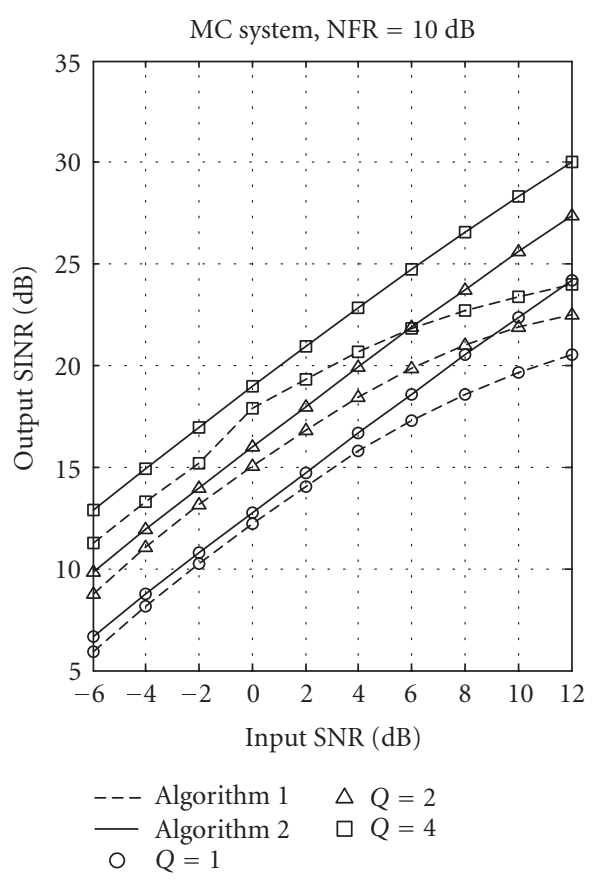

(d) MC system, NFR=10 dB

FIGURE 5: Simulation results (output SINR versus input SNR for low-rate data length $N=2500$ ) obtained by Algorithms 1 (dashed line) and 2 (solid line) using the approach of temporal processing followed by BMRC with $1(\bigcirc), 2(\triangle)$, and $4(\square)$ antennas used.

partly presented at the IEEE Workshop on Signal Processing Advances in Wireless Communications, Lisbon, Portugal, July 11-14, 2004.

\section{REFERENCES}

[1] D. Wong and T. J. Lim, "Soft handoffs in CDMA mobile systems," IEEE Personal Communications, vol. 4, no. 6, pp. 6-17, 1997.
[2] S. Verdu, Multiuser Detection, Cambridge University Press, Cambridge, UK, 1998.

[3] S. Moshavi, "Multi-user detection for DS-CDMA communications," IEEE Communications Magazine, vol. 34, no. 10, pp. 124-136, 1996.

[4] T. Ojanperä and R. Prasad, "An overview of air interface multiple access for IMT-2000/UMTS," IEEE Communications Magazine, vol. 36, no. 9, pp. 82-86, 1998. 
[5] M. Zeng, A. Annamalai, and V. K. Bhargava, "Harmonization of global third-generation mobile systems," IEEE Communications Magazine, vol. 38, no. 12, pp. 94-104, 2000.

[6] C.-Y. Chi, C.-C. Feng, C.-H. Chen, and C.-Y. Chen, Blind Equalization and System Identification, Springer, London, UK, 2006.

[7] C.-Y. Chi, C.-Y. Chen, C.-H. Chen, and C.-C. Feng, "Batch processing algorithms for blind equalization using higherorder statistics," IEEE Signal Processing Magazine, vol. 20, no. 1, pp. 25-49, 2003.

[8] C.-Y. Chi, C.-H. Chen, and C.-Y. Chen, "Blind MAI and ISI suppression for DS/CDMA systems using HOS-based inverse filter criteria," IEEE Transactions on Signal Processing, vol. 50, no. 6, pp. 1368-1381, 2002

[9] C.-L. I and R. D. Gitlin, "Multi-code CDMA wireless personal communications networks," in Proceedings of the IEEE International Conference on Communications, vol. 2, pp. 10601064, Seattle, Wash, USA, June 1995.

[10] T. Ottosson and A. Svensson, "Multi-rate schemes in DS/CDMA systems," in Proceedings of the 45th IEEE Vehicular Technology Conference, vol. 2, pp. 1006-1010, Chicago, Ill, USA, July 1995.

[11] M. K. Tsatsanis, Z. Xu, and X. Lu, "Blind multiuser detectors for dual rate DS-CDMA systems over frequency selective channels," in Proceedings of European Signal Processing Conference, vol. 2, pp. 631-634, Tampere, Finland, September 2000.

[12] S. Roy and H. Yan, "Blind channel estimation in multirate CDMA systems," IEEE Transactions on Communications, vol. 50, no. 6, pp. 995-1004, 2002.

[13] J. Ma and J. K. Tugnait, "Blind detection of multirate asynchronous CDMA signals in multipath channels," IEEE Transactions on Signal Processing, vol. 50, no. 9, pp. 2258-2272, 2002.

[14] Z. Xu and P. Liu, "Code constrained CMA-based multirate multiuser detection," in Proceedings of the Conference Record of the 35th Asilomar Conference on Signals, Systems and Computers, vol. 2, pp. 1455-1459, Pacific Grove, Calif, USA, November 2001.

[15] M. Saquib, R. D. Yates, and A. Ganti, "An asynchronous multirate decorrelator," IEEE Transactions on Communications, vol. 48, no. 5, pp. 739-742, 2000.

[16] M.-H. Chung, K.-C. Chen, and M. Y. You, "MMSE multiuser detection for multi-rate wideband CDMA communications," in Proceedings of 11th IEEE International Symposium on Personal, Indoor and Mobile Radio Communications (PIMRC '00), vol. 1, pp. 534-538, London, UK, September 2000.

[17] U. Mitra, "Comparison of maximum-likelihood-based detection for two multirate access schemes for CDMA signals," IEEE Transactions on Communications, vol. 47, no. 1, pp. 64-77, 1999.

[18] J. G. Proakis, Digital Communications, McGraw-Hill, New York,USA, 4th edition, 2001.

[19] M. K. Tsatsanis and Z. Xu, "Performance analysis of minimum variance CDMA receivers," IEEE Transactions on Signal Processing, vol. 46, no. 11, pp. 3014-3022, 1998.

[20] M. Torlak and G. Xu, "Blind multiuser channel estimation in asynchronous CDMA systems," IEEE Transactions on Signal Processing, vol. 45, no. 1, pp. 137-147, 1997. $-0.5 \mathrm{pt} 2.5 \mathrm{pt}$

[21] S. E. Bensley and B. Aazhang, "Subspace-based channel estimation for code division multiple access communication systems," IEEE Transactions on Communications, vol. 44, no. 8, pp. 1009-1020, 1996.

[22] M. K. Tsatsanis and G. B. Giannakis, "Optimal decorrelating receivers for DS-CDMA systems: a signal processing framework," IEEE Transactions on Signal Processing, vol. 44, no. 12, pp. 3044-3055, 1996. $-0.5 \mathrm{pt} 2.5 \mathrm{pt}$

[23] C.-Y. Chi and C.-H. Chen, "Cumulant-based inverse filter criteria for MIMO blind deconvolution: properties, algorithms, and application to DS/CDMA systems in multipath," IEEE Transactions on Signal Processing, vol. 49, no. 7, pp. 1282-1299, 2001.

[24] X. Wang and H. V. Poor, "Blind equalization and multiuser detection in dispersive CDMA channels," IEEE Transactions on Communications, vol. 46, no. 1, pp. 91-103, 1998.

[25] T. Ristaniemi and J. Joutsensalo, "Advanced ICA-based receivers for block fading DS-CDMA channels," Signal Processing, vol. 82, no. 3, pp. 417-431, 2002.

[26] C.-Y. Chi and C.-Y. Chen, "Blind beamforming and maximum ratio combining by kurtosis maximization for source separation in multipath," in Proceedings of IEEE Workshop on Signal Processing Advances in Wireless Communications, pp. 243-246, Taoyuan, Taiwan, March 2001.

[27] O. Shalvi and E. Weinstein, "Super-exponential methods for blind deconvolution," IEEE Transactions on Information Theory, vol. 39, no. 2, pp. 504-519, 1993.

[28] J. K. Tugnait, "Identification and deconvolution of multichannel linear non-Gaussian processes using higher order statistics and inverse filter criteria," IEEE Transactions on Signal Processing, vol. 45, no. 3, pp. 658-672, 1997.

[29] C.-Y. Chi and C.-H. Peng, "Turbo source extraction algorithm and noncancellation source separation algorithms by kurtosis maximization," IEEE Transactions on Signal Processing, vol. 54, no. 8, pp. 2929-2942, 2006.

[30] C.-H. Peng, C.-Y. Chi, I.-C. Chang, and C.-H. Kuo, "A blind space-time decoding algorithm by kurtosis maximization for the down-link of MC-CDMA systems," in Proceedings of 5th IEEE International Conference on Information, Communications and Signal Processing (ICICS '05), Bangkok, Thailand, December 2005.

Chun-Hsien Peng was born in Taiwan, on November 21, 1978. He received the B.S. degree from the Department of Electronic Engineering, National Taipei University of Technology, Taipei, Taiwan, in 2001, and the Ph.D. degree from the Institute of Communications Engineering and Department of Electrical Engineering, National Tsing Hua University, Hsinchu, Taiwan, in 2006. His research interests include statistical signal processing, digital signal processing, and wireless communications.

Chong-Yung Chi received the Ph.D. degree in electrical engineering from the University of Southern California in 1983. From 1983 to 1988, he was with the Jet Propulsion Laboratory, Pasadena, California. He has been a Professor with the Department of Electrical Engineering since 1989 and the Institute of Communications Engineering (ICE) since 1999 (also the Chairman of ICE for 2002-2005), Na-

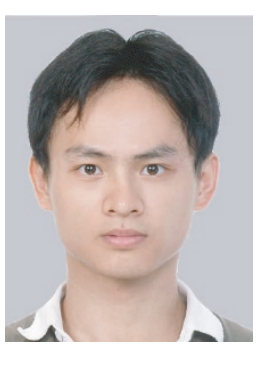
tional Tsing Hua University, Hsinchu, Taiwan. He coauthored a technical book Blind Equalization and System Identification published by Springer 2006, and published more than 120

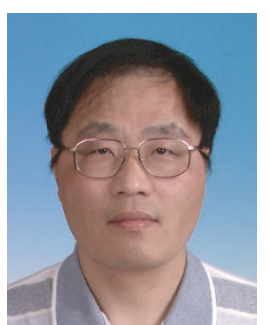


technical papers. His current research interests include signal processing for wireless communications, and statistical signal processing. He is a Senior Member of IEEE. He has been a Technical Program Committee Member for many IEEE-sponsored workshops, symposiums and conferences on signal processing and wireless communications, including co-organizer and general cochairman of IEEE SPAWC 2001. He was an Associate Editor of IEEE Transactions on Signal Processing (May 2001 through April 2006). Currently, he is an Associate Editor for the IEEE Signal Processing Letters, an Associate Editor for the IEEE Transactions on Circuits and Systems II, and a Guest Editor of EURASIP Journal on Applied Signal Processing, a Member of Editorial Board of EURASIP Signal Processing Journal.

Chia-Wen Chang was born in Taiwan, on August 26, 1981. He received the B.S. degree from the Department of Electrical Engineering, Feng Chia University, Taichung, Taiwan, in 2004, and the M.S. degree from the Institute of Communications Engineering and Department of Electrical Engineering, National Tsing Hua University, Hsinchu, Taiwan, in 2006. His research interests include digital signal processing and

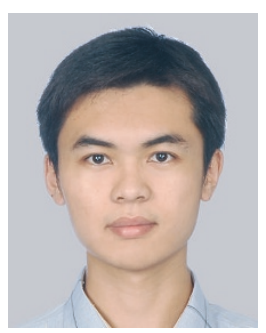
wireless communications. 